\title{
Transmit Optimization with Improper Gaussian Signaling for Interference Channels
}

\author{
Yong Zeng, Student Member, IEEE, Cenk M. Yetis, Member, IEEE, Erry Gunawan, Member, IEEE, \\ Yong Liang Guan, Member, IEEE, and Rui Zhang, Member, IEEE
}

\begin{abstract}
This paper studies the achievable rates of Gaussian interference channels with additive white Gaussian noise (AWGN), when improper or circularly asymmetric complex Gaussian signaling is applied. For the Gaussian multiple-input multiple-output interference channel (MIMO-IC) with the interference treated as Gaussian noise, we show that the user's achievable rate can be expressed as a summation of the rate achievable by the conventional proper or circularly symmetric complex Gaussian signaling in terms of the users' transmit covariance matrices, and an additional term, which is a function of both the users' transmit covariance and pseudo-covariance matrices. The additional degrees of freedom in the pseudocovariance matrix, which is conventionally set to be zero for the case of proper Gaussian signaling, provide an opportunity to further improve the achievable rates of Gaussian MIMO-ICs by employing improper Gaussian signaling. To this end, this paper proposes widely linear precoding, which efficiently maps proper information-bearing signals to improper transmitted signals at each transmitter for any given pair of transmit covariance and pseudo-covariance matrices. In particular, for the case of twouser Gaussian single-input single-output interference channel (SISO-IC), we propose a joint covariance and pseudo-covariance optimization algorithm with improper Gaussian signaling to achieve the Pareto-optimal rates. By utilizing the separable structure of the achievable rate expression, an alternative algorithm with separate covariance and pseudo-covariance optimization is also proposed, which guarantees the rate improvement over conventional proper Gaussian signaling.
\end{abstract}

Index Terms-Improper Gaussian signaling, interference channel, pseudo-covariance optimization, widely linear precoding.

\section{INTRODUCTION}

The interference channel (IC) models multi-user communication systems where each transmitter is intended to send independent information to its corresponding receiver while causing interference to all other receivers. Although informationtheoretic study of the IC has a long history, characterization of its capacity region still remains an open problem in general, except for some special cases such as that with the presence of "strong" interference [1]2]. For the single-input singleoutput IC, termed SISO-IC, the best achievable rate region to date is obtained by the celebrated Han-Kobayashi scheme [2]. Recently, it has been shown in [3] that a particular form of this scheme achieves within one bit to the capacity region of

Y. Zeng, E. Gunawan, and Y. L. Guan are with the School of Electrical and Electronic Engineering (EEE), Nanyang Technological University (NTU), Singapore (e-mail: ze0003ng@e.ntu.edu.sg, \{egunawan, eylguan\}@ntu.edu.sg).

C. M. Yetis was with the School of EEE, NTU. He is now with Mevlana University, Turkey (e-mail: cenkmyetis@gmail.com).

R. Zhang is with the Electrical and Computer Engineering Department, National University of Singapore (e-mail: elezhang@nus.edu.sg). the two-user Gaussian SISO-IC with additive white Gaussian noise (AWGN). Since such capacity-approaching techniques require multi-user encoding/decoding, which are difficult to implement in practical systems, a more pragmatic approach is to employ single-user encoding and decoding by treating the interference as Gaussian noise at all receivers. In fact, this simplified approach has been shown to be sum-capacity optimal for Gaussian ICs when the interference level is below a certain threshold [4].

Under the assumption of single-user detection (SUD) with the interference treated as Gaussian noise, the transmit optimization problem for Gaussian ICs reduces to resource allocation among the transmitters for interference mitigation, which has received significant attention in the last few decades. Early works on resource allocation for Gaussian ICs mostly focused on power control since SISO-IC with single-antenna terminals was considered (see e.g. [5]-[7] and references therein). When transmitters/receivers are equipped with multiple antennas, the network performance can be further improved via transmit/receive beamforming. One useful technique applied for optimizing transmit beamformers is to transform the design problem into an equivalent receiver beamformer optimization problem via the so-called uplink-downlink or network duality principle $[8]-[12]$. The transmit beamforming optimization problems for power minimization and signal-to-interferenceplus-noise ratio (SINR) balancing can also be directly solved by convex optimization techniques, such as the second-order cone programming (SOCP) [13] and semidefinite programming (SDP) [14].

For Gaussian ICs with the interference treated as Gaussian noise, the sum-rate maximization problem is in general difficult to be solved globally optimally due to its non-convexity. In [15], it was shown that finding globally optimal beamformers for the weighted sum-rate maximization (WSRMax) in the Gaussian multiple-input single-output IC (MISO-IC) is an NPhard problem. Algorithms based on the principle of interference pricing have been proposed for achieving local optimums [16], while in [17], a distributed algorithm was proposed for MISO-IC by using the virtual SINR framework. Gradient descent algorithms have also been proposed for Gaussian multiple-input multiple-output IC (MIMO-IC) over transmit covariance matrices [18] or precoding matrices [19]. More recently, for Gaussian SISO-IC, single-input multiple-output IC (SIMO-IC) and MISO-IC, globally optimal solutions to the WSRMax problem have been obtained under the monotonic optimization framework [20]-[24]. However, the complexity of such globally optimal solutions increases exponentially with 
the number of users, and their generalization to more general MIMO-IC remains unknown. An alternative technique for solving WSRMax problems for MIMO-ICs is via iteratively minimizing the weighted mean-square-error (MSE), which utilizes the inherent relationship between the mutual information and MSE [25]-[27]. The study of ICs with game-theoretic models has also been given in [28] and references therein. Moreover, it is worth mentioning that there has been a great deal of interest in the last few years on studying Gaussian ICs from the degrees-of-freedom (DoF) perspective [29]. A key technique to achieve higher DoFs than previously believed for Gaussian ICs is interference alignment (IA) [30]. Since DoF only provides the approximated capacity at the asymptotically high signal-to-noise ratio (SNR), a number of IA-based precoding schemes with improved sum-rate performance at practical SNRs have been proposed in [19], [31]-[33].

As for characterization of the achievable rate region for Gaussian ICs with interference treated as noise, various solutions have been obtained for the SISO-IC [34], SIMO-IC [22], and MISO-IC [35]-[38]. The Pareto boundary of the achievable rate region for ICs consists of all the achievable ratetuples, at each of which it is impossible to improve one user's rate, without simultaneously decreasing the rate of at least one of other users. A traditional approach for characterizing Pareto boundaries of Gaussian ICs is via solving WSRMax problems. However, as pointed out in [36], the WSRMax approach cannot guarantee the finding of all Pareto-boundary points due to the non-convexity of the achievable rate region. An alternative method based on the concept of rate-profile was thus proposed in [36], which is able to characterize the complete Pareto boundary for ICs. Besides, the rate-profile approach generally results in optimization problems that are easier to handle than conventional WSRMax problems for ICs [22[36].

It is necessary to point out that in all the aforementioned works on Gaussian ICs, the transmitted signals are assumed to be proper or circularly symmetric complex Gaussian (CSCG) distributed. A key property of proper Gaussian random vectors (RVs) is that their second-order statistics are completely specified by the conventional covariance matrix under a zeromean assumption. In contrast, for the more general improper Gaussian RVs, an extra parameter called pseudo-covariance matrix is required for the complete second-order characterization [39]-[41]. Most of the existing works on Gaussian ICs have adopted the proper Gaussian assumption without any justification. This may be due to the common practice of modeling the additive receiver noise as proper Gaussian, and the well-known maximum-entropy theorem [39], i.e., proper Gaussian RVs maximize the differential entropy for any given covariance matrix. As a result, proper Gaussian signaling has been shown to be capacity optimal for the Gaussian point-to-point channel, multiple-access channel (MAC) and broadcast channel (BC). However, for Gaussian ICs with the interference treated as noise, improper Gaussian signaling provides a new opportunity to further improve the achievable rates over the conventional proper Gaussian signaling [42]. For instance, it was shown in [43] that improper Gaussian signaling, together with symbol extensions and IA, is able to improve the DoF for the three-user SISO-IC with timeinvariant channel coefficients at the asymptotically high SNR. In [44 45], it was shown that the achievable rate region can be enlarged with improper Gaussian signaling even for the twouser SISO-IC at finite SNR. This is particularly interesting since it is known that for the two-user SISO-IC, no DoF gain is achievable with IA [42]. More specifically, a suboptimal scheme was proposed in [44] for the two-user SISO-IC, where the transmit covariance matrices for the equivalent real-valued MIMO-ICs are restricted to be rank-1. In [45], the Paretooptimal transmit covariance matrices for the two-user SISO-IC are obtained by an exhaustive search method.

The prior works $[43]-[45]$ on the study of improper Gaussian signaling for ICs are all based on the equivalent doublesized real-valued MIMO-IC matrix by separating the real and imaginary parts of the complex-valued channels. Although any complex-valued system can be transformed into an equivalent real-valued system, as pointed out in [40 46], much of the elegancy of the system description is lost. Therefore, in this paper, we adopt the complex-valued channel model for studying improper Gaussian signaling in Gaussian ICs to gain new insights. The main contributions of this paper are summarized as follows:

- Based on existing results on improper Gaussian RVs, we derive a new achievable rate expression for the general $K$-user MIMO-IC, when improper Gaussian signaling is applied. Our result shows that the user's achievable rate can be expressed as a summation of the rate achievable by the conventional proper Gaussian signaling in terms of the users' transmit covariance matrices, and an additional term, which is a function of both the users' transmit covariance and pseudo-covariance matrices. This new result implies that the use of improper Gaussian signaling for MIMO-ICs with interference treated as noise is able to improve the achievable rate over the conventional proper Gaussian signaling with any given set of covariance matrices of transmitted signals, by further optimizing their pseudo-covariance matrices.

- For any given pair of signal covariance and pseudocovariance matrices at each transmitter, we consider the practical problem of generating improper transmitted signals from proper information-bearing signals. Based on existing techniques for improper RVs [46], we propose an efficient method for this implementation, named as widely linear precoding.

- By adopting the rate-profile method, we formulate the optimization problem for the two-user SISO-IC to characterize the Pareto boundary of the achievable rate region with improper Gaussian signaling. By applying the celebrated semidefinite relaxation (SDR) technique [47], a joint covariance and pseudo-covariance optimization algorithm is proposed, which achieves near-optimal ratepairs. Furthermore, by utilizing the separable structure of the achievable rate expression with improper Gaussian signaling, a separate covariance and pseudo-covariance optimization algorithm is also proposed, which guarantees the rate improvement over conventional proper 
Gaussian signaling with any given transmit covariance.

The rest of this paper is organized as follows. Section III studies improper Gaussian signaling for the general MIMOIC, where a new achievable rate expression is derived and widely linear precoding is proposed. Section IIII focuses on the two-user SISO-IC setup, where the problem formulation for characterizing the Pareto boundary of the achievable rate region is given. In Section IV] a SDR-based joint covariance and pseudo-covariance optimization algorithm for the twouser SISO-IC is proposed. In Section V, an alternative SOCPbased algorithm by separate covariance and pseudo-covariance optimizations is presented. Section VI provides numerical results. Finally, we conclude the paper in Section VII.

Notations: In this paper, scalars are denoted by italic letters. Boldface lower- and upper-case letters denote vectors and matrices, respectively. $\mathbf{I}_{M}$ denotes an $M \times M$ identity matrix and the subscript $M$ is omitted if its value is clear from the context. $\mathbf{0}$ denotes an all-zero matrix. For a square matrix $\mathbf{S}, \operatorname{Tr}(\mathbf{S}),|\mathbf{S}|, \mathbf{S}^{-1}$ denote the trace, determinant and inverse of $\mathbf{S}$, respectively. $\mathbf{S} \succeq \mathbf{0}$ and $\mathbf{S} \succ \mathbf{0}$ mean that $\mathbf{S}$ is positive semidefinite and positive definite, respectively. $\mathbb{C}^{M \times N}$ and $\mathbb{R}^{M \times N}$ denote the space of $M \times N$ complex and real matrices, respectively. $\mathbb{S}^{K}$ and $\mathbb{H}^{K}$ denote the $K \times K$ realvalued symmetric and complex-valued Hermitian matrices, respectively. For an arbitrary matrix $\mathbf{A}, \mathbf{A}^{*}, \mathbf{A}^{T}, \mathbf{A}^{H}$ and $\operatorname{rank}\{\mathbf{A}\}$ represent the complex-conjugate, transpose, conjugate transpose and rank of $\mathbf{A}$, respectively. $\operatorname{diag}\{\mathbf{x}\}$ represents a diagonal matrix with the elements in the main diagonal given by $\mathbf{x} . \mathcal{N}(\boldsymbol{\mu}, \mathbf{C})$ represents the real-valued Gaussian RV with mean $\boldsymbol{\mu}$ and covariance matrix $\mathbf{C} . \mathcal{C N}(\mathbf{x}, \boldsymbol{\Sigma})$ represents the CSCG RV with mean $\mathrm{x}$ and covariance matrix $\boldsymbol{\Sigma}$. For a complex number $x,|x|$ denotes its magnitude. The symbol $i$ represents the imaginary unit, i.e., $i^{2}=-1$. $[\mathbf{v}]_{k}$ denotes the $k$ th element of a vector $\mathbf{v} . \Re\{\cdot\}$ and $\Im\{\cdot\}$ represent the real and imaginary parts of complex numbers/matrices, respectively. $I(\mathbf{x} ; \mathbf{y})$ represents the mutual information between two RVs $\mathbf{x}$ and $\mathbf{y}$.

\section{IMPROPER GAUSSIAN SigNALING FOR MIMO-IC}

Consider a $K$-user MIMO-IC, where each transmitter is intended to send independent information to its corresponding receiver, while possibly interfering with all other $K-1$ receivers. Denote the number of transmitting and receiving antennas for each user by $M$ and $N$, respectively. Assuming the narrow-band transmission, the equivalent baseband received signal at each receiver can be expressed as

$$
\mathbf{y}_{k}(n)=\mathbf{H}_{k k} \mathbf{x}_{k}(n)+\sum_{j \neq k} \mathbf{H}_{k j} \mathbf{x}_{j}(n)+\mathbf{n}_{k}(n), \forall k,
$$

where $n$ is the symbol index, $\mathbf{H}_{k k} \in \mathbb{C}^{N \times M}$ denotes the direct channel matrix from transmitter $k$ to receiver $k$, while $\mathbf{H}_{k j}, j \neq k$, denotes the interference channel matrix from transmitter $j$ to receiver $k$; we assume quasi-static fading and thus all channels are constant over $n$ 's in (1) for the case of our interest; $\mathbf{n}_{k}(n)$ represents the independent and identically distributed (i.i.d.) CSCG noise vector at receiver $k$ with $\mathbf{n}_{k}(n) \sim \mathcal{C N}\left(\mathbf{0}, \sigma^{2} \mathbf{I}\right)$; and $\mathbf{x}_{k}(n) \in \mathbb{C}^{M}$ is the transmitted signal vector from transmitter $k$, which is independent of $\mathbf{x}_{j}(n), \forall j \neq k$. In this paper, for the purpose of exposition, we assume that symbol extensions over time as in [43] are not used. Hence, $\mathbf{x}_{k}(n)$ is independent over $n$. For brevity, $n$ is omitted in the rest of this paper. Different from the conventional setup where proper Gaussian signaling is assumed, i.e., $\mathbf{x}_{k} \sim \mathcal{C N}\left(\mathbf{0}, \mathbf{C}_{\mathbf{x}_{k}}\right)$, with $\mathbf{C}_{\mathbf{x}_{k}}$ denoting the transmit covariance matrix, in this paper we consider the more general improper Gaussian signals, for which some preliminaries are given next.

\section{A. Preliminary for Improper Random Vectors}

For a zero-mean $\mathrm{RV} \mathbf{z} \in \mathbb{C}^{n}$, the covariance matrix $\mathbf{C}_{\mathbf{z}}$ and pseudo-covariance matrix $\widetilde{\mathbf{C}}_{z}$ are defined as [39]

$$
\mathbf{C}_{\mathbf{z}} \triangleq \mathbb{E}\left(\mathbf{z z}^{H}\right), \widetilde{\mathbf{C}}_{\mathbf{z}} \triangleq \mathbb{E}\left(\mathbf{z z}^{T}\right) \text {. }
$$

By definition, it is easy to verify that the covariance matrix $\mathbf{C}_{\mathbf{z}}$ is Hermitian and positive semidefinite, and the pseudocovariance matrix $\widetilde{\mathbf{C}}_{\mathbf{z}}$ is symmetric.

Definition 1. [39]: A complex $R V \mathbf{z}$ is called proper if its pseudo-covariance matrix $\widetilde{\mathbf{C}}_{\mathbf{z}}$ vanishes to a zero matrix; otherwise, it is called improper.

Lemma 1. [39]: Two zero-mean complex $R V s \mathrm{x}$ and $\mathbf{z}$ are uncorrelated if and only if $\mathbf{C}_{\mathbf{x z}}=\mathbf{0}$ and $\widetilde{\mathbf{C}}_{\mathbf{x z}}=\mathbf{0}$, where $\mathbf{C}_{\mathbf{x} \mathbf{z}} \triangleq \mathbb{E}\left(\mathbf{x z}^{H}\right)$ and $\widetilde{\mathbf{C}}_{\mathbf{x z}} \triangleq \mathbb{E}\left(\mathbf{x z}^{T}\right)$.

A more restrictive definition than properness is known as circularly symmetric, which is defined as follows.

Definition 2. [40]: A complex $R V \mathrm{z}$ is circularly symmetric if its distribution is rotationally invariant, i.e., $\mathbf{z}$ and $\hat{\mathbf{z}}=e^{i \alpha} \mathbf{z}$ have the same distribution for any real value $\alpha$.

For a circularly symmetric RV z, we have

$$
\widetilde{\mathbf{C}}_{\mathbf{z}}=\widetilde{\mathbf{C}}_{\hat{\mathbf{z}}}=\mathbb{E}\left(\hat{\mathbf{z}}_{\mathbf{z}}^{T}\right)=e^{i 2 \alpha} \widetilde{\mathbf{C}}_{\mathbf{z}}, \forall \alpha,
$$

which implies $\widetilde{\mathbf{C}}_{\mathbf{z}}=\mathbf{0}$. Thus, circularity implies properness, but the converse is not true in general. However, if $\mathbf{z}$ is a zero-mean Gaussian RV, then properness and circularity are equivalent, as given by the following lemma.

Lemma 2. [40]: A complex zero-mean Gaussian $R V \mathbf{z}$ is circularly symmetric if and only if it is proper.

For example, the commonly adopted assumption that the noise vector $\mathbf{n}_{k}$ in (1) is zero-mean CSCG is equivalent to that $\mathbf{n}_{k}$ is a proper Gaussian RV, whose pseudo-covariance matrix satisfies $\mathbf{C}_{\mathbf{n}_{k}}=\mathbf{0}$. For an arbitrary complex RV z, define $\underline{\mathbf{C}}_{\mathbf{z}}$ as the covariance matrix of the augmented vector $\left[\begin{array}{ll}\mathbf{z}^{T} & \left(\mathbf{z}^{*}\right)^{T}\end{array}\right]^{T}$, i.e.,

$$
\underline{\mathbf{C}}_{\mathbf{z}} \triangleq \mathbb{E}\left(\left[\begin{array}{c}
\mathbf{z} \\
\mathbf{z}^{*}
\end{array}\right]\left[\begin{array}{c}
\mathbf{z} \\
\mathbf{z}^{*}
\end{array}\right]^{H}\right)=\left[\begin{array}{ll}
\mathbf{C}_{\mathbf{z}} & \widetilde{\mathbf{C}}_{\mathbf{z}} \\
\widetilde{\mathbf{C}}_{\mathbf{z}}^{*} & \mathbf{C}_{\mathbf{z}}^{*}
\end{array}\right]
$$

The augmented covariance matrix $\underline{\mathbf{C}}_{\mathbf{z}}$ obviously has some built-in redundancy for the second-order characterization of $\mathbf{z}$; however, it is useful as shown in the following two theorems.

Theorem 1. [40]: $\mathbf{C}_{\mathbf{z}}$ and $\widetilde{\mathbf{C}}_{\mathbf{z}}$ are a valid pair of covariance and pseudo-covariance matrices, i.e., there exists a $R V \mathrm{z}$ with covariance and pseudo-covariance matrices given by $\mathbf{C}_{\mathbf{z}}$ and $\widetilde{\mathbf{C}}_{\mathbf{z}}$, respectively, if and only if the augmented covariance matrix $\underline{\mathbf{C}}_{\mathbf{z}}$ is positive semidefinite, i.e., $\underline{\mathbf{C}}_{\mathbf{z}} \succeq \mathbf{0}$. 
Note that the conditions of the covariance matrix $\mathbf{C}_{\mathbf{z}}$ being Hermitian and positive semidefinite, and the pseudocovariance matrix $\widetilde{\mathbf{C}}_{\mathbf{z}}$ being symmetric are already implied by $\mathbf{C}_{\mathbf{z}} \succeq \mathbf{0}$. Furthermore, for the improper complex Gaussian $\mathrm{RVs}$, the differential entropy is in general a function of both the covariance and pseudo-covariance matrices, which can be expressed in terms of $\underline{\mathbf{C}}_{\mathbf{z}}$ as shown by the following theorem.

Theorem 2. [40]: The entropy of a complex Gaussian $R V$ $\mathbf{z} \in \mathbb{C}^{n}$ with augmented covariance matrix $\underline{\mathbf{C}}_{\mathbf{z}}$ is

$$
h(\mathbf{z})=\frac{1}{2} \log \left((\pi e)^{2 n}\left|\underline{\mathbf{C}}_{\mathbf{z}}\right|\right) .
$$

Theorem 2 generalizes the entropy result for proper Gaussian RVs. If $\mathbf{C}_{\mathbf{z}}=\mathbf{0}$, (4) reduces to the well-known expression for proper Gaussian RVs $h(\mathbf{z})=\log \left((\pi e)^{n}\left|\mathbf{C}_{\mathbf{z}}\right|\right)$ [39].

\section{B. Achievable Rate with Improper Gaussian Signaling}

In this subsection, we derive the achievable rate by improper Gaussian signaling for the $K$-user MIMO-IC defined in (1). Denote the covariance and pseudo-covariance matrices of the zero-mean transmitted Gaussian RV $\mathbf{x}_{k}$ by $\mathbf{C}_{\mathbf{x}_{k}}$ and $\widetilde{\mathbf{C}}_{\mathbf{x}_{k}}$, respectively, i.e.,

$$
\mathbf{C}_{\mathbf{x}_{k}}=\mathbb{E}\left(\mathbf{x}_{k} \mathbf{x}_{k}^{H}\right), \widetilde{\mathbf{C}}_{\mathbf{x}_{k}}=\mathbb{E}\left(\mathbf{x}_{k} \mathbf{x}_{k}^{T}\right), k=1, \cdots, K .
$$

Since $\mathbf{x}_{k}$ and $\mathbf{x}_{j}$ are independent for $j \neq k$, then by using Lemma 1 and the fact that independence and uncorrelatedness are equivalent for Gaussian RVs, the covariance and pseudocovariance matrices of the received signal vector $\mathbf{y}_{k}, k=$ $1, \cdots, K$, can be obtained as

$$
\begin{aligned}
& \mathbf{C}_{\mathbf{y}_{k}}=\mathbb{E}\left(\mathbf{y}_{k} \mathbf{y}_{k}^{H}\right)=\sum_{j=1}^{K} \mathbf{H}_{k j} \mathbf{C}_{\mathbf{x}_{j}} \mathbf{H}_{k j}^{H}+\sigma^{2} \mathbf{I}, \\
& \widetilde{\mathbf{C}}_{\mathbf{y}_{k}}=\mathbb{E}\left(\mathbf{y}_{k} \mathbf{y}_{k}^{T}\right)=\sum_{j=1}^{K} \mathbf{H}_{k j} \widetilde{\mathbf{C}}_{\mathbf{x}_{j}} \mathbf{H}_{k j}^{T},
\end{aligned}
$$

where in (6), we have used the fact that the pseudo-covariance of the CSCG noise vector $\mathbf{n}_{k}$ is a zero matrix. It is obvious from (5) that $\mathbf{C}_{\mathbf{y}_{k}}$ is nonsingular. Then with the augmented covariance matrix $\underline{\mathbf{C}}_{\mathbf{y}_{k}}$ defined as in (3) and using the Schur complement [48], we obtain

$$
\begin{aligned}
\left|\underline{\mathbf{C}}_{\mathbf{y}_{k}}\right| & =\left|\begin{array}{ll}
\widetilde{\mathbf{C}}_{\mathbf{y}_{k}} & \widetilde{\mathbf{C}}_{\mathbf{y}_{k}}^{*} \\
\mathbf{y}_{k} & \mathbf{C}_{\mathbf{y}_{k}}^{*}
\end{array}\right|=\left|\mathbf{C}_{\mathbf{y}_{k}}\right|\left|\mathbf{C}_{\mathbf{y}_{k}}^{*}-\widetilde{\mathbf{C}}_{\mathbf{y}_{k}}^{*} \mathbf{C}_{\mathbf{y}_{k}}^{-1} \widetilde{\mathbf{C}}_{\mathbf{y}_{k}}\right| \\
& =\left|\mathbf{C}_{\mathbf{y}_{k}}\right|^{2}\left|\mathbf{I}-\mathbf{C}_{\mathbf{y}_{k}}^{-1} \widetilde{\mathbf{C}}_{\mathbf{y}_{k}} \mathbf{C}_{\mathbf{y}_{k}}^{-T} \widetilde{\mathbf{C}}_{\mathbf{y}_{k}}^{H}\right|,
\end{aligned}
$$

where we have used the fact that for an invertible matrix $\mathbf{A}$, $\mathbf{A}^{-T}=\left(\mathbf{A}^{-1}\right)^{T}=\left(\mathbf{A}^{T}\right)^{-1}$; and in the last equality, we have used the identities $|\mathbf{A}|=\left|\mathbf{A}^{T}\right|,|\mathbf{A B}|=|\mathbf{A}||\mathbf{B}|$, and the facts that $\mathbf{C}_{\mathbf{y}_{k}}$ is Hermitian and $\mathbf{C}_{\mathbf{y}_{k}}$ is symmetric. With the transmitted signals being Gaussian, the received signal $\mathbf{y}_{k}$ is also Gaussian. Then based on Theorem 2, the differential entropy of $\mathbf{y}_{k} \in \mathbb{C}^{N}$ is given by

$$
h\left(\mathbf{y}_{k}\right)=\log \left((\pi e)^{N}\left|\mathbf{C}_{\mathbf{y}_{k}}\right|\right)+\frac{1}{2} \log \left|\mathbf{I}-\mathbf{C}_{\mathbf{y}_{k}}^{-1} \widetilde{\mathbf{C}}_{\mathbf{y}_{k}} \mathbf{C}_{\mathbf{y}_{k}}^{-T} \widetilde{\mathbf{C}}_{\mathbf{y}_{k}}^{H}\right| .
$$

Denote $\mathbf{s}_{k}$ as the interference-plus-noise term at receiver $k$, i.e., $\mathbf{s}_{k}=\sum_{j \neq k} \mathbf{H}_{k j} \mathbf{x}_{j}+\mathbf{n}_{k}$. Then the covariance and pseudocovariance matrices of $\mathbf{s}_{k}$ are given by

$$
\begin{aligned}
\mathbf{C}_{\mathbf{s}_{k}} & =\sum_{j \neq k} \mathbf{H}_{k j} \mathbf{C}_{\mathbf{x}_{j}} \mathbf{H}_{k j}^{H}+\sigma^{2} \mathbf{I}, \\
\widetilde{\mathbf{C}}_{\mathbf{s}_{k}} & =\sum_{j \neq k} \mathbf{H}_{k j} \widetilde{\mathbf{C}}_{\mathbf{x}_{j}} \mathbf{H}_{k j}^{T} .
\end{aligned}
$$

Similarly as for $\mathbf{y}_{k}$, the differential entropy of $\mathbf{s}_{k}$ is

$$
h\left(\mathbf{s}_{k}\right)=\log \left((\pi e)^{N}\left|\mathbf{C}_{\mathbf{s}_{k}}\right|\right)+\frac{1}{2} \log \left|\mathbf{I}-\mathbf{C}_{\mathbf{s}_{k}}^{-1} \widetilde{\mathbf{C}}_{\mathbf{s}_{k}} \mathbf{C}_{\mathbf{s}_{k}}^{-T} \widetilde{\mathbf{C}}_{\mathbf{s}_{k}}^{H}\right| .
$$

Under the assumption that interference is treated as Gaussian noise, the achievable rate at receiver $k$ with improper Gaussian signaling can be obtained as

$$
\begin{array}{r}
R_{k}=I\left(\mathbf{x}_{k} ; \mathbf{y}_{k}\right)=h\left(\mathbf{y}_{k}\right)-h\left(\mathbf{y}_{k} \mid \mathbf{x}_{k}\right)=h\left(\mathbf{y}_{k}\right)-h\left(\mathbf{s}_{k}\right) \\
=\frac{1}{2} \log \frac{\left|\underline{\mathbf{C}}_{\mathbf{y}_{k}}\right|}{\left|\underline{\mathbf{C}}_{\mathbf{s}_{k}}\right|}= \\
=\underbrace{\log \frac{\left|\sigma^{2} \mathbf{I}+\sum_{j=1}^{K} \mathbf{H}_{k j} \mathbf{C}_{\mathbf{x}_{j}} \mathbf{H}_{k j}^{H}\right|}{\left|\sigma^{2} \mathbf{I}+\sum_{j \neq k} \mathbf{H}_{k j} \mathbf{C}_{\mathbf{x}_{j}} \mathbf{H}_{k j}^{H}\right|}}_{\triangleq R_{k, \text { proper }}\left(\left\{\mathbf{C}_{\mathbf{x}_{j}}\right\}\right)} \\
+\frac{1}{2} \log \frac{\left|\mathbf{I}-\mathbf{C}_{\mathbf{y}_{k}}^{-1} \widetilde{\mathbf{C}}_{\mathbf{y}_{k}} \mathbf{C}_{\mathbf{y}_{k}}^{-T} \widetilde{\mathbf{C}}_{\mathbf{y}_{k}}^{H}\right|}{\left|\mathbf{I}-\mathbf{C}_{\mathbf{s}_{k}}^{-1} \widetilde{\mathbf{C}}_{\mathbf{s}_{k}} \mathbf{C}_{\mathbf{s}_{k}}^{-T} \widetilde{\mathbf{C}}_{\mathbf{s}_{k}}^{H}\right|} .
\end{array}
$$

The above equation shows that with improper Gaussian signaling, the achievable rate can be expressed as a summation of two terms. The first term, denoted by $R_{k, \text { proper }}\left(\left\{\mathbf{C}_{\mathbf{x}_{j}}\right\}\right)$, is the rate achievable by the conventional proper Gaussian signaling, which is a function of the transmit covariance matrices only. The second term is a function of both the transmit covariance and pseudo-covariance matrices. By setting $\widetilde{\mathbf{C}}_{\mathbf{x}_{k}}=\mathbf{0}, \forall k$, the second term vanishes and $(10)$ reduces to the rate expression for the conventional case of proper Gaussian signaling. The separability of the achievable rate by improper Gaussian signaling provides a general method to improve the achievable rate over the conventional proper Gaussian signaling, i.e., for any given covariance matrices obtained by existing proper Gaussian signaling schemes, the rate can be improved with improper Gaussian signaling by choosing the pseudocovariance matrices that make the second term in (10) strictly positive. It is worth noting that this property does not exist if we convert the complex-valued system in (1) to an equivalent real-valued system by doubling the input/output dimensions.

In this paper, we are interested in characterizing the achievable rate region with improper Gaussian signaling. The achievable rate region for the $K$-user MIMO-IC consists of all the rate-tuples for all users that can be simultaneously achieved under a given set of transmit power constraints for each transmitter, denoted by $P_{k}, k=1, \ldots, K$, i.e.,

$$
\mathcal{R} \triangleq \bigcup_{\substack{\operatorname{Tr}\left\{\mathbf{C}_{\mathbf{x}_{\mathbf{k}}}\right\} \leq P_{k} \\ \underline{\mathbf{C}}_{\mathbf{x}_{k}} \succeq \mathbf{0}, \forall k}}\left\{\left(r_{1}, \cdots, r_{K}\right): 0 \leq r_{k} \leq R_{k}, \forall k\right\},
$$

where $\underline{\mathbf{C}}_{\mathbf{x}_{k}}$ is the augmented covariance matrix of $\mathbf{x}_{k}$ defined in (3). The constraint $\underline{\mathbf{C}}_{\mathbf{x}_{k}} \succeq \mathbf{0}$ follows from Theorem 1 In Sections [II V], we will consider the transmit covariance and pseudo-covariance optimizations for achieving the Pareto boundary of the above rate region for the special case of twouser SISO-IC.

\section{Widely Linear Precoding}

In this subsection, we consider the practical problem of how to efficiently generate the transmitted signal $\mathbf{x}_{k}$ at each transmitter given any valid pair of covariance matrix $\mathbf{C}_{\mathbf{x}_{k}}$ and pseudo-covariance matrix $\mathbf{C}_{\mathbf{x}_{k}}$, from an information-bearing signal $\mathbf{d}_{k}$ that is selected from conventional CSCG (proper Gaussian) codebooks. Without loss of generality, we assume $\mathbf{d}_{k} \sim \mathcal{C N}(\mathbf{0}, \mathbf{I})$; thus, we have

$$
\mathbf{C}_{\mathbf{d}_{k}}=\mathbf{I}, \widetilde{\mathbf{C}}_{\mathbf{d}_{k}}=\mathbf{0}, k=1, \cdots, K .
$$


First, consider the conventional linear precoding given by

$$
\mathbf{x}_{k}=\mathbf{U}_{k} \mathbf{d}_{k} \text {, }
$$

where $\mathbf{U}_{k}$ is the precoding matrix. Then the pseudo-covariance matrix of $\mathbf{x}_{k}$ is given by $\widetilde{\mathbf{C}}_{\mathbf{x}_{k}}=\mathbf{U}_{k} \widetilde{\mathbf{C}}_{\mathbf{d}_{k}} \mathbf{U}_{k}^{T}=\mathbf{0}$. This implies that the conventional linear precoding is not able to map the proper Gaussian signal $\mathbf{d}_{k}$ to the improper transmitted Gaussian signal $\mathbf{x}_{k}$.

Since the augmented covariance matrix defined in (3) contains both the covariance and pseudo-covariance matrices, a necessary condition for a RV $\mathbf{z}_{k}$ to have covariance matrix $\mathbf{C}_{\mathbf{x}_{k}}$ and pseudo-covariance matrices $\widetilde{\mathbf{C}}_{\mathbf{x}_{k}}$ is that its augmented covariance matrix satisfies $\underline{\mathbf{C}}_{\mathbf{z}_{k}}=\underline{\mathbf{C}}_{\mathbf{x}_{k}}$. This is ensured by the transformation

$$
\left[\begin{array}{l}
\mathbf{z}_{k} \\
\mathbf{z}_{k}^{*}
\end{array}\right]=\underline{\mathbf{C}}_{\mathbf{x}_{k}}^{\frac{1}{2}}\left[\begin{array}{l}
\mathbf{d}_{k} \\
\mathbf{d}_{k}^{*}
\end{array}\right]
$$

where $\underline{\mathbf{C}}_{\mathbf{x}_{k}}^{\frac{1}{2}}$ denotes the generalized Cholesky factor of the positive semidefinite matrix $\underline{\mathbf{C}}_{\mathbf{x}_{k}}$, which is defined by $\underline{\mathbf{C}}_{\mathbf{x}_{k}}=$ $\underline{\mathbf{C}}_{\mathbf{x}_{k}}^{\frac{1}{2}}\left(\underline{\mathbf{C}}_{\mathbf{x}_{k}}^{\frac{1}{2}}\right)^{H}$. Since $\underline{\mathbf{C}}_{\mathbf{d}_{k}}=\mathbf{I}$ as given in (12), it is easy to verify that $\mathbf{z}_{k}$ in (14) satisfies $\underline{\mathbf{C}}_{\mathbf{z}_{k}}=\underline{\mathbf{C}}_{\mathbf{x}_{k}}$. A common method for finding $\underline{\mathbf{C}}_{\mathbf{x}_{k}}^{\frac{1}{2}}$ is via eigenvalue decomposition (EVD). Specifically, let the EVD of $\underline{\mathbf{C}}_{\mathbf{x}_{k}}$ be expressed as $\underline{\mathbf{C}}_{\mathbf{x}_{k}}=\mathbf{U D U}{ }^{H}$; then $\underline{\mathbf{C}}_{\mathbf{x}_{k}}^{\frac{1}{2}}=\mathbf{U} \mathbf{D}^{\frac{1}{2}}$ is obtained. However, it is worth pointing out that the above obtained $\underline{\mathbf{C}}_{\mathbf{x}_{k}}^{\frac{1}{2}}$ cannot satisfy (14) in general. This is because the two vectors $\mathbf{z}_{k}$ and $\mathbf{z}_{k}^{*}$ in (14) need to be complex conjugate of each other; therefore, the transformation matrix $\underline{\mathbf{C}}_{\mathbf{x}_{k}}^{\frac{1}{2}}$ should be designed with more care than the conventional EVD. On the other hand, if we can find one $\underline{\mathbf{C}}_{\mathbf{x}_{k}}^{\frac{1}{2}}$ such that it has the following structure:

$$
\underline{\mathbf{C}}_{\mathbf{x}}^{\frac{1}{2}}=\left[\begin{array}{ll}
\mathbf{B}_{1} & \mathbf{B}_{2} \\
\mathbf{B}_{2}^{*} & \mathbf{B}_{1}^{*}
\end{array}\right]
$$

i.e., the upper-left (w.r.t. upper-right) block is the complex conjugate of the lower-right (w.r.t. lower-left) block, then (14) is equivalent to the following two sets of equations:

$$
\begin{aligned}
& \mathbf{z}_{k}=\mathbf{B}_{1} \mathbf{d}_{k}+\mathbf{B}_{2} \mathbf{d}_{k}^{*}, \\
& \mathbf{z}_{k}^{*}=\mathbf{B}_{2}^{*} \mathbf{d}_{k}+\mathbf{B}_{1}^{*} \mathbf{d}_{k}^{*} .
\end{aligned}
$$

It is easy to verify that the two equations given in 16 and (17) are consistent, i.e., (17) is simply obtained by taking the complex conjugate on both sides of 16 and vice versa. Therefore, the remaining task is to find one $\underline{\mathbf{C}}_{\mathbf{x}}^{\frac{1}{2}}$ with the structure given by (15). To achieve this end, we define the following $2 M \times 2 M$ unitary matrix [46]:

$$
\mathbf{T} \triangleq \frac{1}{\sqrt{2}}\left[\begin{array}{cc}
\mathbf{I}_{M} & i \mathbf{I}_{M} \\
\mathbf{I}_{M} & -i \mathbf{I}_{M}
\end{array}\right], \quad \mathbf{T} \mathbf{T}^{H}=\mathbf{T}^{H} \mathbf{T}=\mathbf{I}_{2 M} .
$$

For any real-valued matrix $\mathbf{A}=\left[\begin{array}{ll}\mathbf{A}_{11} & \mathbf{A}_{12} \\ \mathbf{A}_{21} & \mathbf{A}_{22}\end{array}\right] \in \mathbb{R}^{2 M \times 2 M}$, it can be verified that the matrix $\mathbf{T A} \mathbf{T}^{H}$ has the structure given in (15), i.e.,

$$
\mathbf{T A T}^{H}=\left[\begin{array}{ll}
\mathbf{A}_{1} & \mathbf{A}_{2} \\
\mathbf{A}_{2}^{*} & \mathbf{A}_{1}^{*}
\end{array}\right]
$$

with $\mathbf{A}_{1}=\frac{1}{2}\left[\left(\mathbf{A}_{11}+\mathbf{A}_{22}\right)+i\left(\mathbf{A}_{21}-\mathbf{A}_{12}\right)\right]$ and $\mathbf{A}_{2}=$ $\frac{1}{2}\left[\left(\mathbf{A}_{11}-\mathbf{A}_{22}\right)+i\left(\mathbf{A}_{21}+\mathbf{A}_{12}\right)\right]$.

Theorem 3. [46] There exists one form of EVD for the augmented covariance matrix $\underline{\mathbf{C}}_{\mathbf{x}_{k}} \in \mathbb{C}^{2 M \times 2 M}$ defined in (3), which is given by

$$
\underline{\mathbf{C}}_{\mathbf{x}_{k}}=(\mathbf{T V}) \boldsymbol{\Lambda}(\mathbf{T V})^{H},
$$

where $\mathbf{V} \in \mathbb{R}^{2 M \times 2 M}{ }^{\frac{\mathbf{C}_{\mathbf{x}_{k}}}{2}}$ is a real-valued orthogonal matrix and
$\boldsymbol{\Lambda}=\operatorname{diag}\left\{\lambda_{1}, \lambda_{2}, \cdots, \lambda_{2 M}\right\}$ contains the eigenvalues of $\underline{\mathbf{C}}_{\mathbf{x}_{k}}$.

In fact, (19) can be obtained by considering $\mathbf{T}^{H} \underline{\mathbf{C}}_{\mathbf{x}_{k}} \mathbf{T}$, which is a real-valued matrix given by

$$
\mathbf{T}^{H} \underline{\mathbf{C}}_{\mathbf{x}_{k}} \mathbf{T}=\left[\begin{array}{ll}
\Re\left\{\mathbf{C}_{\mathbf{x}_{k}}+\widetilde{\mathbf{C}}_{\mathbf{x}_{k}}\right\} & \Im\left\{-\mathbf{C}_{\mathbf{x}_{k}}+\widetilde{\mathbf{C}}_{\mathbf{x}_{k}}\right\} \\
\Im\left\{\mathbf{C}_{\mathbf{x}_{k}}+\widetilde{\mathbf{C}}_{\mathbf{x}_{k}}\right\} & \Re\left\{\mathbf{C}_{\mathbf{x}_{k}}-\widetilde{\mathbf{C}}_{\mathbf{x}_{k}}\right\}
\end{array}\right] .
$$

Furthermore, since $\mathbf{C}_{\mathbf{x}_{k}}$ is Hermitian and $\widetilde{\mathbf{C}}_{\mathbf{x}_{k}}$ is symmetric, it can be verified that the matrix $\mathbf{T}^{H} \underline{\mathbf{C}}_{\mathbf{x}_{k}} \mathbf{T}$ is symmetric as well. Therefore, its real-valued EVD can be written as

$$
\mathbf{T}^{H} \underline{\mathbf{C}}_{\mathbf{x}_{k}} \mathbf{T}=\mathbf{V} \boldsymbol{\Lambda} \mathbf{V}^{T}
$$

The EVD in (19) is then obtained by applying a unitary transformation $\mathbf{T}$ to 20 .

For any given $\underline{\mathbf{C}}_{\mathbf{x}_{k}} \succeq \mathbf{0}$, all the eigenvalues are nonnegative [46], i.e., $\lambda_{l} \geq 0, l=1, \cdots, 2 M$. Thus (19) can be written as $\underline{\mathbf{C}}_{\mathbf{x}_{k}}=\mathbf{T V} \boldsymbol{\Lambda} \mathbf{V}^{H} \mathbf{T}^{H}=\left(\mathbf{T V} \boldsymbol{\Lambda}^{1 / 2} \mathbf{T}^{H}\right)\left(\mathbf{T V} \boldsymbol{\Lambda}^{1 / 2} \mathbf{T}^{H}\right)^{H}$.

Then we have

$$
\underline{\mathbf{C}}_{\mathbf{x}_{k}}^{\frac{1}{2}}=\mathbf{T}\left(\mathbf{V} \boldsymbol{\Lambda}^{1 / 2}\right) \mathbf{T}^{H}=\left[\begin{array}{ll}
\mathbf{B}_{1} & \mathbf{B}_{2} \\
\mathbf{B}_{2}^{*} & \mathbf{B}_{1}^{*}
\end{array}\right],
$$

where the last equality follows from (18) and the fact that $\mathbf{V} \boldsymbol{\Lambda}^{1 / 2}$ is a real-valued matrix.

From (14) and 21), it follows that to obtain the transmitted signal vector $\mathrm{x}_{k}$, which is generally improper with the covariance and pseudo-covariance matrices specified by $\underline{\mathbf{C}}_{\mathbf{x}_{k}}$, the following precoding needs to be applied to the proper information-bearing signal $\mathbf{d}_{k}$ :

$$
\mathbf{x}_{k}=\mathbf{B}_{1} \mathbf{d}_{k}+\mathbf{B}_{2} \mathbf{d}_{k}^{*}
$$

where $\mathbf{B}_{1}$ and $\mathbf{B}_{2}$ are the corresponding blocks in $\mathbf{T V} \boldsymbol{\Lambda}^{1 / 2} \mathbf{T}^{H}$ as shown in (21), with $\mathbf{V}$ and $\boldsymbol{\Lambda}$ obtained by the particular form of EVD in (19). Following similar terminologies used in existing literatures on improper signal processing such as [49], we refer to the precoding given in 22) as widely linear precoding. Note that if $\mathbf{B}_{2}=\mathbf{0}$, which is the case when $\underline{\mathbf{C}}_{\mathbf{x}_{k}}$ is block-diagonal (i.e., $\widetilde{\mathbf{C}}_{\mathbf{x}_{k}}=\mathbf{0}$ ), 22) reduces to the conventional linear precoding for proper Gaussian signaling given by 13 . Last, in terms of the realvalued representation, 22 can be re-expressed as

$$
\left[\begin{array}{l}
\Re\left\{\mathbf{x}_{k}\right\} \\
\Im\left\{\mathbf{x}_{k}\right\}
\end{array}\right]=\left[\begin{array}{ll}
\Re\left\{\mathbf{B}_{1}+\mathbf{B}_{2}\right\} & \Im\left\{\mathbf{B}_{2}-\mathbf{B}_{1}\right\} \\
\Im\left\{\mathbf{B}_{2}+\mathbf{B}_{1}\right\} & \Re\left\{\mathbf{B}_{1}-\mathbf{B}_{2}\right\}
\end{array}\right]\left[\begin{array}{l}
\Re\left\{\mathbf{d}_{k}\right\} \\
\Im\left\{\mathbf{d}_{k}\right\}
\end{array}\right] .
$$

\section{Pareto Boundary Characterization for the TWO-USER SISO-IC}

In the remaining part of this paper, we will focus on the special two-user SISO-IC case, with the aim of characterizing its Pareto rate boundary with improper Gaussian signaling by optimizing both the covariances and pseudo-covariances of transmitted signals. The input-output relationship for the twouser SISO-IC can be simplified from (1) as

$$
\begin{aligned}
& y_{1}=h_{11} x_{1}+h_{12} x_{2}+n_{1}, \\
& y_{2}=h_{21} x_{1}+h_{22} x_{2}+n_{2},
\end{aligned}
$$

where $h_{k j}=\left|h_{k j}\right| e^{i \phi_{k j}}, k, j \in\{1,2\}$, is the complex scalar channel from transmitter $j$ to receiver $k$ 1 Denote the covariance and pseudo-covariance of the transmitted signal $x_{k}$ by

$$
C_{x_{k}}=\mathbb{E}\left(x_{k} x_{k}^{*}\right), \widetilde{C}_{x_{k}}=\mathbb{E}\left(x_{k} x_{k}\right), k=1,2 .
$$

\footnotetext{
${ }^{1}$ Since a phase rotation can be applied at each of the receivers with coherent demodulation, without loss of generality, the direct channel gains $h_{11}$ and $h_{22}$ can be assumed to be real values. However, this assumption will not change the remaining results in this paper.
} 
Note that $C_{x_{k}}$ 's are nonnegative real numbers equal to the transmit power values of the corresponding users, while $\widetilde{C}_{x_{k}}$ 's are complex numbers in general. Due to Schur complement, the necessary and sufficient conditions stated in Theorem 1 for the special case of two-user SISO-IC reduce to

$$
C_{x_{k}} \geq 0,\left|\widetilde{C}_{x_{k}}\right|^{2} \leq C_{x_{k}}^{2}, k=1,2 \text {. }
$$

The covariance and pseudo-covariance of $y_{k}$ can be written as

$$
\begin{aligned}
& C_{y_{k}}=\mathbb{E}\left(y_{k} y_{k}^{*}\right)=\left|h_{k 1}\right|^{2} C_{x_{1}}+\left|h_{k 2}\right|^{2} C_{x_{2}}+\sigma^{2}, \\
& \widetilde{C}_{y_{k}}=\mathbb{E}\left(y_{k} y_{k}\right)=h_{k 1}^{2} \widetilde{C}_{x_{1}}+h_{k 2}^{2} \widetilde{C}_{x_{2}} .
\end{aligned}
$$

For the interference-plus-noise term $s_{k}=h_{k \bar{k}} x_{\bar{k}}+n_{k}, \bar{k} \neq k$, we have

$$
C_{s_{k}}=\left|h_{k \bar{k}}\right|^{2} C_{x_{\bar{k}}}+\sigma^{2}, \widetilde{C}_{s_{k}}=h_{k \bar{k}}^{2} \widetilde{C}_{x_{\bar{k}}} .
$$

Then the achievable rate expression in $(10)$ for the special case of two-user SISO-IC reduces to

$$
\begin{aligned}
& R_{k}^{\text {SISO }}=\frac{1}{2} \log \frac{C_{y_{k}}^{2}-\left|\widetilde{C}_{y_{k}}\right|^{2}}{C_{s_{k}}^{2}-\left|\widetilde{C}_{s_{k}}\right|^{2}} \\
& =\underbrace{\log \left(1+\frac{\left|h_{k k}\right|^{2} C_{x_{k}}}{\sigma^{2}+\left|h_{k \bar{k}}\right|^{2} C_{x_{\bar{k}}}}\right)}_{\triangleq R_{k, \text { proper }}^{\text {SIso }}\left(C_{x_{1}}, C_{x_{2}}\right)}+\frac{1}{2} \log \frac{1-C_{y_{k}}^{-2}\left|\widetilde{C}_{y_{k}}\right|^{2}}{1-C_{s_{k}}^{-2}\left|\widetilde{C}_{s_{k}}\right|^{2}} .
\end{aligned}
$$

To characterize the Pareto boundary of the achievable rate region defined in 11, we adopt the rate-profile technique proposed in [36]. Specifically, any Pareto-optimal rate-pair can be obtained by solving the following optimization problem with a given rate-profile specified by $\boldsymbol{\alpha}=\left(\alpha_{1}, \alpha_{2}\right)$ :

$$
\begin{aligned}
& \text { (P1): } \max _{\left\{C_{x_{k}}\right\},\left\{\widetilde{C}_{x_{k}}\right\}, R} R \\
& \text { s.t. } \quad R_{k}^{\mathrm{SISO}} \geq \alpha_{k} R, \forall k \\
& \quad 0 \leq C_{x_{k}} \leq P_{k}, \forall k \\
&\left|\widetilde{C}_{x_{k}}\right|^{2} \leq C_{x_{k}}^{2}, \forall k,
\end{aligned}
$$

where $\alpha_{k}$ denotes the target ratio between user $k$ 's achievable rate and the users' sum-rate, $R$. Without loss of generality, we assume that $\alpha_{1}, \alpha_{2}>0$ and $\alpha_{1}+\alpha_{2}=1$. Denote the optimal solution to $(\mathrm{P} 1)$ as $R^{\star}$, then the rate-pair $\left(\alpha_{1} R^{\star}, \alpha_{2} R^{\star}\right)$ must be on the Pareto boundary corresponding to the rate-profile given by $\left(\alpha_{1}, \alpha_{2}\right)$. Thereby, by solving (P1) with different rateprofile parameters $\left(\alpha_{1}, \alpha_{2}\right)$, the complete Pareto boundary for the achievable rate region can be found.

\section{JoInt COVARIANCE AND PSEUdo-COVARIANCE OPTIMIZATION}

In this section, by applying the SDR technique, we propose an approximate solution to the non-convex problem (P1) where the covariance and pseudo-covariance of the transmitted signals are jointly optimized. The approach of using SDR for solving non-convex quadratically constrained quadratic program (QCQP) has been successfully applied to find highquality approximate solutions for various problems in communication and signal processing (see e.g. [47] and references therein). By treating $R$ as a slack variable and substituting $R_{k}^{\mathrm{SISO}}$ in 29), (P1) can be equivalently written as

$$
\begin{gathered}
\text { (P1.1): } \max _{\left\{C_{x_{k}}\right\},\left\{\tilde{C}_{x_{k}}\right\}} \min _{k=1,2} \frac{1}{2 \alpha_{k}} \log \frac{C_{y_{k}}^{2}-\left|\widetilde{C}_{y_{k}}\right|^{2}}{C_{s_{k}}^{2}-\left|\widetilde{C}_{s_{k}}\right|^{2}} \\
\text { s.t. } \quad 0 \leq C_{x_{k}} \leq P_{k}, \quad \forall k \\
\left|\widetilde{C}_{x_{k}}\right|^{2} \leq C_{x_{k}}^{2}, \quad \forall k .
\end{gathered}
$$

(P1.1) is a minimum-weighted-rate maximization (MinWRMax) problem, where the weights are related to the rate-profile $\boldsymbol{\alpha}$. The following result will be used for solving (P1.1).

Lemma 3. For any set of $\left\{C_{x_{k}}\right\}$ and $\left\{\widetilde{C}_{x_{k}}\right\}$ that is feasible to (P1.1), the following inequalities hold:

$$
\begin{aligned}
& C_{y_{k}}^{2}-\left|\widetilde{C}_{y_{k}}\right|^{2} \geq \sigma^{4}>0, \quad \forall k, \\
& C_{s_{k}}^{2}-\left|\widetilde{C}_{s_{k}}\right|^{2} \geq \sigma^{4}>0, \quad \forall k .
\end{aligned}
$$

Proof: Please refer to Appendix A

Define the following 2-dimensional real-valued vectors:

$$
\begin{aligned}
& \mathbf{c}=\left[\begin{array}{ll}
C_{x_{1}} & C_{x_{2}}
\end{array}\right]^{T}, \\
& \mathbf{a}_{1}=\left[\begin{array}{ll}
\left|h_{11}\right|^{2} & \left|h_{12}\right|^{2}
\end{array}\right]^{T}, \quad \mathbf{b}_{1}=\left[\begin{array}{ll}
0 & \left|h_{12}\right|^{2}
\end{array}\right]^{T}, \\
& \mathbf{a}_{2}=\left[\begin{array}{ll}
\left|h_{21}\right|^{2} & \left|h_{22}\right|^{2}
\end{array}\right]^{T}, \quad \mathbf{b}_{2}=\left[\begin{array}{ll}
\left|h_{21}\right|^{2} & 0
\end{array}\right]^{T} .
\end{aligned}
$$

Then from (26) and (28), we have

$$
C_{y_{k}}^{2}=\left(\sigma^{2}+\mathbf{a}_{k}^{T} \mathbf{c}\right)^{2}, C_{s_{k}}^{2}=\left(\sigma^{2}+\mathbf{b}_{k}^{T} \mathbf{c}\right)^{2}, k=1,2 .
$$

Define the following 2-dimensional complex-valued vectors:

$$
\begin{aligned}
& \mathbf{q}=\left[\begin{array}{ll}
\widetilde{C}_{x_{1}} & \widetilde{C}_{x_{2}}
\end{array}\right]^{T}, \\
& \mathbf{f}_{1}=\left[\begin{array}{ll}
h_{11}^{2} & h_{12}^{2}
\end{array}\right]^{H}, \mathbf{g}_{1}=\left[\begin{array}{ll}
0 & h_{12}^{2}
\end{array}\right]^{H}, \\
& \mathbf{f}_{2}=\left[\begin{array}{ll}
h_{21}^{2} & h_{22}^{2}
\end{array}\right]^{H}, \mathbf{g}_{2}=\left[\begin{array}{ll}
h_{21}^{2} & 0
\end{array}\right]^{H} .
\end{aligned}
$$

Then from 27) and (28), we have

$$
\begin{aligned}
& \left|\widetilde{C}_{y_{k}}\right|^{2}=\left|\mathbf{f}_{k}^{H} \mathbf{q}\right|^{2}=\mathbf{q}^{H} \mathbf{F}_{k} \mathbf{q}, \\
& \left|\widetilde{C}_{s_{k}}\right|^{2}=\left|\mathbf{g}_{k}^{H} \mathbf{q}\right|^{2}=\mathbf{q}^{H} \mathbf{G}_{k} \mathbf{q},
\end{aligned}
$$

where $\mathbf{F}_{k}=\mathbf{f}_{k} \mathbf{f}_{k}^{H}$ and $\mathbf{G}_{k}=\mathbf{g}_{k} \mathbf{g}_{k}^{H}, k=1,2$. By substituting (38)-(40) into (P1.1), we obtain the following equivalent problem

$$
\begin{aligned}
\text { (P1.2): } \max _{\mathbf{c} \in \mathbb{R}^{2}, \mathbf{q} \in \mathbb{C}^{2}} & \min _{k} \cdot \frac{1}{2 \alpha_{k}} \log \frac{\left(\sigma^{2}+\mathbf{a}_{k}^{T} \mathbf{c}\right)^{2}-\mathbf{q}^{H} \mathbf{F}_{k} \mathbf{q}}{\left(\sigma^{2}+\mathbf{b}_{k}^{T} \mathbf{c}\right)^{2}-\mathbf{q}^{H} \mathbf{G}_{k} \mathbf{q}} \\
\text { s.t. } \quad & \mathbf{c}^{T} \mathbf{E}_{k} \mathbf{c} \leq P_{k}^{2}, \quad \forall k \\
& \mathbf{e}_{k}^{T} \mathbf{c} \geq 0, \quad \forall k \\
& \mathbf{q}^{H} \mathbf{E}_{k} \mathbf{q} \leq \mathbf{c}^{T} \mathbf{E}_{k} \mathbf{c}, \quad \forall k
\end{aligned}
$$

where $\mathbf{e}_{k}$ is the $k$ th column in the identity matrix $\mathbf{I}_{2}$, and $\mathbf{E}_{k}=\mathbf{e}_{k} \mathbf{e}_{k}^{T}$. (41) and (42) correspond to the constraints (34) in (P1.1), and (43) is equivalent to 35). The objective function of (P1.2) is given by the minimum of weighted log-fraction of quadratic functions over $\mathbf{c}$ and $\mathbf{q}$. Due to the noise power $\sigma^{2}$, the quadratics are non-homogeneous [47]. By introducing a new variable $t$, we obtain the homogenized quadratics [47], which yield

$$
\begin{aligned}
\text { (P1.3): } \max _{\mathbf{c}, \mathbf{q}, t} . & \min _{k} \cdot \frac{1}{2 \alpha_{k}} \log \frac{\left(\sigma^{2} t+\mathbf{a}_{k}^{T} \mathbf{c}\right)^{2}-\mathbf{q}^{H} \mathbf{F}_{k} \mathbf{q}}{\left(\sigma^{2} t+\mathbf{b}_{k}^{T} \mathbf{c}\right)^{2}-\mathbf{q}^{H} \mathbf{G}_{k} \mathbf{q}} \\
\text { s.t. } \quad & \mathbf{c}^{T} \mathbf{E}_{k} \mathbf{c} \leq P_{k}^{2}, \quad \forall k \\
& \mathbf{e}_{k}^{T} \mathbf{c} t \geq 0, \quad \forall k \\
& \mathbf{q}^{H} \mathbf{E}_{k} \mathbf{q} \leq \mathbf{c}^{T} \mathbf{E}_{k} \mathbf{c}, \quad \forall k \\
& t^{2}=1 .
\end{aligned}
$$

(P1.3) is equivalent to $(\mathrm{P} 1.2)$ in the sense that if it has an optimal solution $\left(\mathbf{c}^{\star}, \mathbf{q}^{\star}, t^{\star}\right)$, then $\left(\mathbf{c}^{\star} / t^{\star}, \mathbf{q}^{\star} / t^{\star}\right)$ is an optimal solution to (P1.2) with the same optimal value. Therefore, (P1.2) can be solved by solving (P1.3). Next, we show that the celebrated SDR technique can be applied to find an approximate solution to (P1.3). Define

$$
\mathbf{C}=\left[\begin{array}{l}
t \\
\mathbf{c}
\end{array}\right]\left[\begin{array}{l}
t \\
\mathbf{c}
\end{array}\right]^{T}, \mathbf{Q}=\mathbf{q q}^{H},
$$




$$
\begin{aligned}
\mathbf{A}_{k} & =\left[\begin{array}{c}
\sigma^{2} \\
\mathbf{a}_{k}
\end{array}\right]\left[\begin{array}{c}
\sigma^{2} \\
\mathbf{a}_{k}
\end{array}\right]^{T}, \mathbf{B}_{k}=\left[\begin{array}{c}
\sigma^{2} \\
\mathbf{b}_{k}
\end{array}\right]\left[\begin{array}{c}
\sigma^{2} \\
\mathbf{b}_{k}
\end{array}\right]^{T} \\
\mathbf{K}_{k} & =\left[\begin{array}{cc}
0 & \frac{1}{2} \mathbf{e}_{k}^{T} \\
\frac{1}{2} \mathbf{e}_{k} & \mathbf{0}
\end{array}\right], \hat{\mathbf{E}}_{k}=\left[\begin{array}{cc}
0 & \mathbf{0} \\
\mathbf{0} & \mathbf{E}_{k}
\end{array}\right] .
\end{aligned}
$$

With the identity $\mathbf{x}^{H} \mathbf{A} \mathbf{x}=\operatorname{Tr}\left(\mathbf{A} \mathbf{x} \mathbf{x}^{H}\right),(\mathrm{P} 1.3)$ is recast as

$$
\begin{aligned}
\text { (P1.4): } \max _{\mathbf{C} \in \mathbb{S}^{3}, \mathbf{Q} \in \mathbb{H}^{2}} & \min _{k} \cdot \frac{1}{2 \alpha_{k}} \log \frac{\operatorname{Tr}\left(\mathbf{A}_{k} \mathbf{C}\right)-\operatorname{Tr}\left(\mathbf{F}_{k} \mathbf{Q}\right)}{\operatorname{Tr}\left(\mathbf{B}_{k} \mathbf{C}\right)-\operatorname{Tr}\left(\mathbf{G}_{k} \mathbf{Q}\right)} \\
\text { s.t. } \quad & \operatorname{Tr}\left(\hat{\mathbf{E}}_{k} \mathbf{C}\right) \leq P_{k}^{2}, \quad \forall k \\
& \operatorname{Tr}\left(\mathbf{K}_{k} \mathbf{C}\right) \geq 0, \quad \forall k \\
& \operatorname{Tr}\left(\mathbf{E}_{k} \mathbf{Q}\right) \leq \operatorname{Tr}\left(\hat{\mathbf{E}}_{k} \mathbf{C}\right), \quad \forall k \\
& \mathbf{C}_{11}=1 \\
& \mathbf{C} \succeq \mathbf{0}, \mathbf{Q} \succeq \mathbf{0} \\
& \operatorname{rank}(\mathbf{C})=1, \operatorname{rank}(\mathbf{Q})=1
\end{aligned}
$$

where $\mathbf{C}_{11}$ denotes the $(1,1)$-th entry of $\mathbf{C}$; the positive semidefinite constraints (55) and the rank-1 constraints (56) are due to (48). With such a reformulation, the objective function of (P1.4) is now a log-fraction of linear functions of $\mathbf{C}$ and $\mathbf{Q}$, and all the constraints (51)-(54) are also linear. With Lemma 3 and the equivalence between (P1.1) and (P1.4), for any pair of $\mathbf{C}$ and $\mathbf{Q}$ that is feasible to (P1.4), we have

$$
\begin{aligned}
& \operatorname{Tr}\left(\mathbf{A}_{k} \mathbf{C}\right)-\operatorname{Tr}\left(\mathbf{F}_{k} \mathbf{Q}\right) \geq \sigma^{4}>0, \quad \forall k, \\
& \operatorname{Tr}\left(\mathbf{B}_{k} \mathbf{C}\right)-\operatorname{Tr}\left(\mathbf{G}_{k} \mathbf{Q}\right) \geq \sigma^{4}>0, \quad \forall k .
\end{aligned}
$$

The SDR problem of (P1.4) is obtained by discarding the non-convex rank-1 constraints in (56), and including the extra constraints (57) and (58), i.e.,

$$
\begin{gathered}
\text { (P1.4-SDR): } \max _{\mathbf{C} \in \mathbb{S}^{3}, \mathbf{Q} \in \mathbb{H}^{2}} \min _{k} \cdot \frac{1}{2 \alpha_{k}} \log \frac{\operatorname{Tr}\left(\mathbf{A}_{k} \mathbf{C}\right)-\operatorname{Tr}\left(\mathbf{F}_{k} \mathbf{Q}\right)}{\operatorname{Tr}\left(\mathbf{B}_{k} \mathbf{C}\right)-\operatorname{Tr}\left(\mathbf{G}_{k} \mathbf{Q}\right)} \\
\text { s.t. 51]- 55, 57, 58. }
\end{gathered}
$$

Note that although the constraints (57) and (58) are redundant in the rank-constrained problem (P1.4), in the rank-relaxed problem (P1.4-SDR), the advantages of including them are twofold. First, it makes (P1.4-SDR) a problem with less relaxation to (P1.4). Besides, the strict positivity of (58) makes (P1.4-SDR) a quasi-convex problem and hence can be solved with the standard bisection method [50]. Since any feasible solution of (P1.4) is feasible for (P1.4-SDR), the optimal objective value of (P1.4-SDR) provides an upper bound on that of (P1.4). To solve the quasi-convex problem (P1.4-SDR), consider the following feasibility problem for a fixed $R$ :

(P1.5): find $\mathbf{C} \in \mathbb{S}^{3}, \mathbf{Q} \in \mathbb{H}^{2}$

$$
\begin{aligned}
& \text { s.t. } 51 \text { - 55], } 57, \text { (58), } \\
& \operatorname{Tr}\left(\mathbf{A}_{k} \mathbf{C}\right)-\operatorname{Tr}\left(\mathbf{F}_{k} \mathbf{Q}\right) \\
& \geq e^{2 \alpha_{k} R}\left(\operatorname{Tr}\left(\mathbf{B}_{k} \mathbf{C}\right)-\operatorname{Tr}\left(\mathbf{G}_{k} \mathbf{Q}\right)\right), \forall k .
\end{aligned}
$$

(P1.5) is a SDP problem, which can be efficiently solved [50]. If (P1.5) is feasible, then the optimal objective value $R_{\text {sdr }}$ of (P1.4-SDR) satisfies $R_{\text {sdr }} \geq R$; otherwise $R_{\text {sdr }}<R$. Therefore, (P1.4-SDR) can be solved by solving the SDP problem (P1.5) together with a bisection search over $R$.

Denote the solution to (P1.4-SDR) by $\left(\mathbf{C}^{\star}, \mathbf{Q}^{\star}\right)$. If $\operatorname{rank}\left(\mathbf{C}^{\star}\right)=1$ and $\operatorname{rank}\left(\mathbf{Q}^{\star}\right)=1$, then $\left(\mathbf{C}^{\star}, \mathbf{Q}^{\star}\right)$ is also the optimal solution to the rank-constrained problem (P1.4). In this case, SDR is tight and the optimal solution to (P1.3) is given by the principal components of $\mathbf{C}^{\star}$ and $\mathbf{Q}^{\star}$, from which the solution to (P1.2) can be obtained; otherwise, we apply the following Gaussian randomization procedure customized to our problem to obtain an approximate solution to (P1.2) [47].

Algorithm 1 Gaussian Randomization Procedure for (P1.2)

Input: The solution $\left(\mathbf{C}^{\star}, \mathbf{Q}^{\star}\right)$ to (P1.4-SDR) and the number of randomization trials $L$.

$$
\begin{aligned}
& \text { 1: for } l=1, \cdots, L \text { do } \\
& \text { 2: Generate }\left[\begin{array}{l}
t_{l} \\
\boldsymbol{\xi}_{l}
\end{array}\right] \sim \mathcal{N}\left(\mathbf{0}, \mathbf{C}^{\star}\right), \boldsymbol{\beta}_{l} \sim \mathcal{C N}\left(\mathbf{0}, \mathbf{Q}^{\star}\right) \text {. } \\
& \text { 3: } \quad \text { Let } \mathbf{c}_{l}=\boldsymbol{\xi}_{l} / t_{l} \text {, and } \mathbf{q}_{l}=\boldsymbol{\beta}_{l} / t_{l} \text {. } \\
& \text { 4: } \quad \text { Construct a feasible point }\left(\check{\mathbf{c}}_{l}, \check{\mathbf{q}}_{l}\right) \text { for (P1.2) as follows: } \\
& {\left[\check{\mathbf{c}}_{l}\right]_{k}=\max \left(0, \min \left(\left[\mathbf{c}_{l}\right]_{k}, P_{k}\right)\right) \text {, }} \\
& {\left[\check{\mathbf{q}}_{l}\right]_{k}=\eta_{k}\left[\mathbf{q}_{l}\right]_{k} \text {, }} \\
& \text { where } \eta_{k}=\min \left\{1, \frac{\left[\check{\mathbf{c}}_{l}\right]_{k}}{\left|\left[\mathbf{q}_{l}\right]_{k}\right|}\right\}, k=1,2 \text {. } \\
& \max _{\check{\mathbf{c}}_{l}, \check{\mathbf{q}}_{l}, l=1, \cdots, L} \min _{k} \cdot \frac{1}{2 \alpha_{k}} \log \frac{\left(\sigma^{2}+\mathbf{a}_{k}^{T} \check{\mathbf{c}}_{l}\right)^{2}-\check{\mathbf{q}}_{l}^{H} \mathbf{F}_{k} \check{\mathbf{q}}_{l}}{\left(\sigma^{2}+\mathbf{b}_{k}^{T} \check{\mathbf{c}}_{l}\right)^{2}-\check{\mathbf{q}}_{l}^{H} \mathbf{G}_{k} \check{\mathbf{q}}_{l}}
\end{aligned}
$$

\section{5: end for}

Output: $(\hat{\mathbf{c}}, \hat{\mathbf{q}})$ as an approximate solution for (P1.2).

\section{Separate Covariance and Pseudo-Covariance OPTIMIZATION}

For the algorithm proposed in the preceding section, although joint optimizations are performed over the covariances and pseudo-covariances, it is not clear whether a rate gain over conventional proper Gaussian signaling is attainable since the obtained solutions are not necessarily globally optimal. In this section, by utilizing the result that the user's achievable rate is separable as shown in (30), we propose a separate covariance and pseudo-covariance optimization algorithm for (P1). Specifically, the covariances of the transmitted signals are first optimized by setting the pseudo-covariances to be zero, i.e., proper Gaussian signaling is applied. Then, the pseudocovariances are optimized with the covariances fixed as the previously optimized values. With such a separation approach, the obtained improper signaling scheme is guaranteed to improve the rate over proper Gaussian signaling scheme.

\section{A. Covariance Optimization}

When restricted to proper Gaussian signaling with $\widetilde{C}_{x_{1}}=0$ and $\widetilde{C}_{x_{2}}=0$, by substituting (30) into [31), (P1) reduces to

$$
\begin{array}{cl}
\text { (P1.6): } \max _{r, C_{x_{1}}, C_{x_{2}}} & r \\
\text { s.t. } & \log \left(1+\frac{\left|h_{11}\right|^{2} C_{x_{1}}}{\sigma^{2}+\left|h_{12}\right|^{2} C_{x_{2}}}\right) \geq \alpha_{1} r, \\
& \log \left(1+\frac{\left|h_{22}\right|^{2} C_{x_{2}}}{\sigma^{2}+\left|h_{21}\right|^{2} C_{x_{1}}}\right) \geq \alpha_{2} r, \\
& 0 \leq C_{x_{1}} \leq P_{1}, 0 \leq C_{x_{2}} \leq P_{2} .
\end{array}
$$

For any fixed value $r$, (P1.6) can be transformed to the following feasibility problem:

$$
\begin{aligned}
\text { (P1.7): Find } & C_{x_{1}} \in \mathbb{R}, C_{x_{2}} \in \mathbb{R} \\
\text { s.t. } \quad & \left|h_{11}\right|^{2} C_{x_{1}} \geq\left(\sigma^{2}+\left|h_{12}\right|^{2} C_{x_{2}}\right)\left(e^{\alpha_{1} r}-1\right), \\
& \left|h_{22}\right|^{2} C_{x_{2}} \geq\left(\sigma^{2}+\left|h_{21}\right|^{2} C_{x_{1}}\right)\left(e^{\alpha_{2} r}-1\right), \\
& 0 \leq C_{x_{1}} \leq P_{1}, \quad 0 \leq C_{x_{2}} \leq P_{2} .
\end{aligned}
$$


(P1.7) is a linear programming (LP) problem, which can be efficiently solved [50]. If $r$ is feasible to (P1.7), then the optimal value of (P1.6) satisfies $r^{\star} \geq r$; otherwise, $r^{\star}<r$. Thus, (P1.6) can be efficiently solved by solving (P1.7) together with the bisection method for updating $r$.

\section{B. Pseudo-Covariance Optimization}

Denote the optimal solution to the covariance optimization problem (P1.6) as $\left\{r^{\star}, C_{x_{1}}^{\star}, C_{x_{2}}^{\star}\right\}$. By fixing the covariances as $C_{x_{1}}^{\star}$ and $C_{x_{2}}^{\star}$, (P1) is then optimized over the pseudocovariances $\widetilde{C}_{x_{1}}$ and $\widetilde{C}_{x_{2}}$. By substituting the first term in the rate expression (30) with $\alpha_{k} r^{\star}$, the problem for pseudocovariance optimization is formulated as

(P1.8): $\underset{\widetilde{C}_{x_{1}}, \tilde{C}_{x_{2}}, \quad R}{\max }$

$$
\begin{aligned}
\widetilde{C}_{x_{1}}, \widetilde{C}_{x_{2}}, R & \\
\text { s.t. } & \alpha_{1} r^{\star}+\frac{1}{2} \log \frac{1-C_{y_{1}}^{-2}\left|\widetilde{C}_{y_{1}}\right|^{2}}{1-C_{s_{1}}^{-2}\left|\widetilde{C}_{s_{1}}\right|^{2}} \geq \alpha_{1} R, \\
& \alpha_{2} r^{\star}+\frac{1}{2} \log \frac{1-C_{y_{2}}^{-2}\left|\widetilde{C}_{y_{2}}\right|^{2}}{1-C_{s_{2}}^{-2}\left|\widetilde{C}_{s_{2}}\right|^{2}} \geq \alpha_{2} R, \\
& \left|\widetilde{C}_{x_{1}}\right|^{2} \leq C_{x_{1}}^{\star 2},\left|\widetilde{C}_{x_{2}}\right|^{2} \leq C_{x_{2}}^{\star 2},
\end{aligned}
$$

where $C_{y_{1}}, C_{s_{1}}, C_{y_{2}}$ and $C_{s_{2}}$ are the corresponding covariance terms with the transmit covariances $C_{x_{1}}^{\star}$ and $C_{x_{2}}^{\star}$. Again, if a given $R$ is achievable for certain pair of $\widetilde{C}_{x_{1}}$ and $\widetilde{C}_{x_{2}}$, then the optimal value of (P1.8) satisfies $R^{\star} \geq R$; otherwise, $R^{\star}<R$. Therefore, (P1.8) can be solved via solving a set of feasibility problems together with the bisection method. It can be easily observed that $\left\{\widetilde{C}_{x_{1}}=0, \widetilde{C}_{x_{2}}=0, R=r^{\star}\right\}$ is feasible to (P1.8). Therefore, $R^{\star} \geq r^{\star}$ is satisfied, i.e., with our proposed separate covariance and pseudo-covariance optimizations, the users' sum-rate corresponding to the rate-profile given by $\left(\alpha_{1}, \alpha_{2}\right)$ with improper Gaussian signaling is guaranteed to be no smaller than that obtained with the optimal proper Gaussian signaling obtained by solving (P1.6). Therefore, the remaining problem to be solved is the feasibility problem resulting from (P1.8) for a given $R$. By substituting $\widetilde{C}_{y_{k}}$ in 27) and $\widetilde{C}_{s_{k}}$ in (28) into (P1.8) and after some manipulations, the feasibility problem for a given $R$ can be formulated as

(P1.9): Find $\widetilde{C}_{x_{1}} \in \mathbb{C}, \widetilde{C}_{x_{2}} \in \mathbb{C}$

$$
\begin{array}{ll}
\text { s.t. } & a_{1}\left|h_{11}^{2} \widetilde{C}_{x_{1}}+h_{12}^{2} \widetilde{C}_{x_{2}}\right|^{2}+b_{1} \leq\left|\widetilde{C}_{x_{2}}\right|^{2}, \\
& a_{2}\left|h_{21}^{2} \widetilde{C}_{x_{1}}+h_{22}^{2} \widetilde{C}_{x_{2}}\right|^{2}+b_{2} \leq\left|\widetilde{C}_{x_{1}}\right|^{2}, \\
& \left|\widetilde{C}_{x_{1}}\right|^{2} \leq C_{x_{1}}^{\star 2}, \\
& \left|\widetilde{C}_{x_{2}}\right|^{2} \leq C_{x_{2}}^{\star 2}
\end{array}
$$

where $a_{k}=\frac{C_{s_{k}}^{2}}{\beta_{k} C_{y_{k}}^{2}\left|h_{k \bar{k}}\right|^{4}}, b_{k}=\frac{\left(1-1 / \beta_{k}\right) C_{s_{k}}^{2}}{\left|h_{k \bar{k}}\right|^{4}}, \beta_{k}=e^{2 \alpha_{k}\left(R-r^{\star}\right)}$, $k=1,2, \vec{k} \neq k$. Since the optimal value of (P1.8) satisfies $R^{\star} \geq r^{\star}$, we can assume that $R \geq r^{\star}$ in (P1.9) without loss of optimality. Then it follows that $\beta_{k} \geq 1, b_{k} \geq 0, \forall k$. In the following, we show that (P1.9) can be efficiently solved via solving a finite number of SOCP problems. First, it can be verified that if $\left\{\widetilde{C}_{x_{1}}, \widetilde{C}_{x_{2}}\right\}$ is feasible for (P1.9), then so is $\left\{\widetilde{C}_{x_{1}} e^{i \omega}, \widetilde{C}_{x_{2}} e^{i \omega}\right\}$. Therefore, without loss of generality, we may choose the common phase rotation $\omega$ so that $\widetilde{C}_{x_{1}}$ is real and nonnegative. Denote the magnitude and phase of $\widetilde{C}_{x_{2}}$ by $t$ and $\theta$, respectively, i.e., $\widetilde{C}_{x_{2}}=t e^{i \theta}$. Then for any fixed

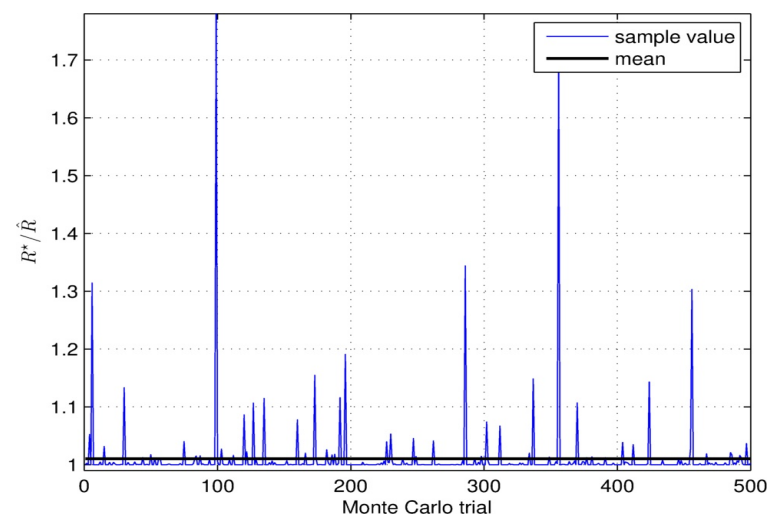

Fig. 1: Empirical ratio $R^{\star} / \hat{R}$ for the two-user SISO-IC over 500 random channel realizations, and with $\mathrm{SNR}=0 \mathrm{~dB}$.

value of $\theta$, (P1.9) can be transformed into a SOCP feasibility problem given by

$$
\begin{array}{cl}
\text { (P1.10): Find } & \widetilde{C}_{x_{1}} \in \mathbb{R}, t \in \mathbb{R} \\
\text { s.t. } & \left\|\sqrt{a_{1}}\left(h_{11}^{2} \widetilde{C}_{x_{1}}+h_{12}^{2} t e^{i \theta}\right)\right\| \leq t, \\
& \sqrt{b_{1}}
\end{array}
$$

Theorem 4. The feasibility problem (P1.9) can be optimally solved by solving a finite number of SOCP problems (P1.10), each for a fixed value $\theta$, where $\theta$ can be restricted to the following discrete set:

$\theta \in\left\{\pi+2\left(\phi_{11}-\phi_{12}\right), \pi+2\left(\phi_{21}-\phi_{22}\right)\right\} \cup \Theta_{\mathcal{A}} \cup \Theta_{\mathcal{B}}$, where $\Theta_{\mathcal{A}}$ and $\Theta_{\mathcal{B}}$ are the solution sets for $\theta$ to the following two sets of equations with variables $(\theta, t)$ and $\left(\theta, \widetilde{C}_{x_{1}}\right)$, respectively:

$$
\begin{gathered}
\Theta_{\mathcal{A}}:\left\{\begin{array}{l}
a_{1}\left|h_{11}^{2} C_{x_{1}}^{\star}+h_{12}^{2} t e^{i \theta}\right|^{2}+b_{1}=t^{2} \\
a_{2}\left|h_{21}^{2} C_{x_{1}}^{\star}+h_{22}^{2} t e^{i \theta}\right|^{2}+b_{2}=C_{x_{1}}^{\star 2}
\end{array}\right. \\
\Theta_{\mathcal{B}}:\left\{\begin{array}{l}
a_{1}\left|h_{11}^{2} \widetilde{C}_{x_{1}}+h_{12}^{2} C_{x_{2}}^{\star} e^{i \theta}\right|^{2}+b_{1}=C_{x_{2}}^{\star 2} \\
a_{2}\left|h_{21}^{2} \widetilde{C}_{x_{1}}+h_{22}^{2} C_{x_{2}}^{\star} e^{i \theta}\right|^{2}+b_{2}=\widetilde{C}_{x_{1}}^{2}
\end{array}\right.
\end{gathered}
$$

Proof: Please refer to Appendix B.

Theorem 4 can be intuitively explained as follows. For the feasibility problem (P1.9), if the constraint $(62)$ is more "restrictive" than (63), then $\theta$ should have a value such that the left hand side (LHS) of (62) is minimized. This corresponds to $\theta=\pi+2\left(\phi_{11}-\phi_{12}\right)$ so that $h_{11}^{2} \widetilde{C}_{x_{1}}$ and $h_{12}^{2} \widetilde{C}_{x_{2}}$ are antiphase. Similar argument for $\theta=\pi+2\left(\phi_{21}-\phi_{22}\right)$ can be made. On the other hand, if both (62) and (63) are equally "restrictive", a feasible solution tends to make both constraints satisfied with equality, as given by 66 and 67$). \Theta_{\mathcal{A}}$ and $\Theta_{\mathcal{B}}$ correspond to the cases where either the constraint (64) or 65) is active, which can be assumed without loss of generality as shown by Proposition 1 in Appendix B. The elements in $\Theta_{\mathcal{A}}$ and $\Theta_{\mathcal{B}}$ can be efficiently obtained by following the steps in Appendix C

\section{Numerical Results}

In this section, we evaluate the performance of the proposed algorithms for the two-user SISO-IC with numerical exam- 


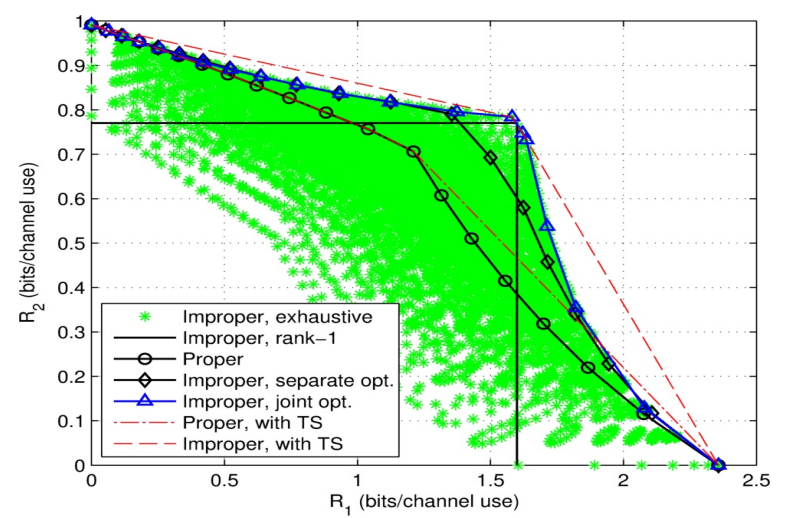

Fig. 2: Achievable rate region for the two-user SISO-IC with channel realization $\mathbf{H}^{(1)}$, and $\mathrm{SNR}=0 \mathrm{~dB}$.

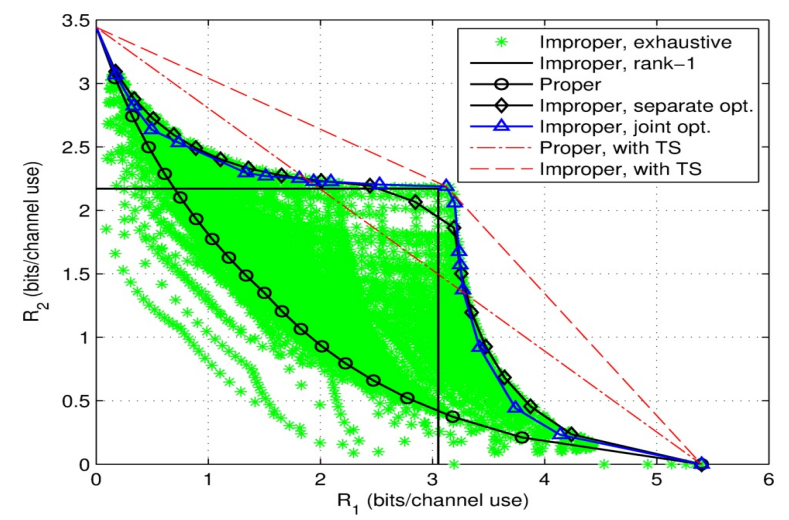

Fig. 3: Achievable rate region for the two-user SISO-IC with channel realization $\mathbf{H}^{(1)}$, and $\mathrm{SNR}=10 \mathrm{~dB}$.

ples. Both transmitters are assumed to have the same power constraint $P$, i.e., $P_{1}=P_{2}=P$. SNR is defined as $P / \sigma^{2}$. For the SDR-based joint covariance and pseudo-covariance optimization algorithm, $L=1000$ is used for the Gaussian randomization procedure in Algorithm 1 .

\section{A. Approximation Ratio for SDR}

In this subsection, we evaluate the quality of the approximate solution obtained by the SDR-based joint covariance and pseudo-covariance optimization algorithm proposed in Section [V] Denote $R^{\star}$ and $R_{\text {sdr }}$ as the optimal objective values of (P1.4) and its relaxation (P1.4-SDR), respectively. Further denote $\hat{R}$ as the objective value of (P1.2) corresponding to the approximate solution obtained by Algorithm 1 . Then

$$
\hat{R} \leq R^{\star} \leq R_{\text {sdr }}, \text { or } 1 \leq R^{\star} / \hat{R} \leq R_{\text {sdr }} / \hat{R},
$$

where $R^{\star} / \hat{R}$ is the approximation ratio. Since in general the optimal value $R^{\star}$ is difficult to be found, the upper bound $R_{\mathrm{sdr}} / \hat{R}$ of the approximation ratio is usually used to evaluate the quality of the obtained approximate solutions [47]. Fortunately, for the two-user SISO-IC considered herein, the optimal value $R^{\star}$ of (P1.1) and hence that of its equivalent problem (P1.4)) can be obtained by the exhaustive search method proposed in [45]. With the rate-profile $\alpha$ setting to $(1 / 2,1 / 2)$, the empirical ratios of $R^{\star} / \hat{R}$ over 500 random channel realizations at $\mathrm{SNR}=0 \mathrm{~dB}$ are plotted in Fig. 1. where the channel coefficients are generated from i.i.d. CSCG random variables with zero-mean and unit-variance. It is found that the mean of $R^{\star} / \hat{R}$ is 1.01 , which demonstrates the high quality of the approximate solution obtained by the SDRbased joint covariance and pseudo-covariance optimization algorithm.

\section{B. Rate Region Comparison}

In Fig. 2 and Fig. 3 , the achievable rate regions for an example two-user SISO-IC are plotted for SNR $=0 \mathrm{~dB}$ and 10 $\mathrm{dB}$, respectively. The channel matrix for both plots is given by $\mathbf{H}^{(1)}=\left[\begin{array}{ll}h_{11} & h_{12} \\ h_{21} & h_{22}\end{array}\right]=\left[\begin{array}{cc}2.0310 e^{-i 0.6858} & 1.4766 e^{i 2.6452} \\ 0.7280 e^{i 1.9726} & 0.9935 e^{-i 0.6676}\end{array}\right]$. The proposed improper Gaussian signaling schemes with joint and separate covariance and pseudo-covariance optimizations are compared with other existing schemes, including the optimal proper Gaussian signaling scheme by solving (P1.6), the optimal improper Gaussian signaling obtained by the exhaustive search method [45], and the rank-1 improper Gaussian signaling scheme [44]. Both figures reveal that for the given channel $\mathbf{H}^{(1)}$, the achievable rate regions are significantly enlarged with improper Gaussian signaling over the conventional proper Gaussian signaling. The plots also demonstrate that the SDR-based joint covariance and pseudo-covariance optimization algorithm yields almost the optimal rates given by the exhaustive search, which is consistent with the observation in Fig. 1. Moreover, it is observed that the separate covariance and pseudo-covariance optimization algorithm performs close to the optimal solution, and also always outperforms the optimal proper signaling. It is worth remarking that, even with time-sharing (TS) ${ }^{2}$ improper Gaussian signaling still outperforms proper Gaussian signaling, as shown by the dashed lines in the two figures. For this particular channel realization, the Pareto boundary points of the achievable rate region with TS using improper Gaussian signaling can be obtained by the TS between the two single-user maximum rate points, and the largest rate corner point by the existing rank-1 scheme [44]. However, this is not always the case, as illustrated by the next example.

Next, consider a two-user SISO-IC channel given by $\mathbf{H}^{(2)}=$ $\left[\begin{array}{cc}4.0 e^{i 1.7730} & 0.90 e^{i 1.6744} \\ 0.80 e^{i 0.6249} & 1.50 e^{i 2.1057}\end{array}\right]$. It is observed from Fig. 4 that for this particular channel realization at $S N R=0 \mathrm{~dB}$, there is no notable performance gain by using improper Gaussian signaling over proper signaling, which is in contrast to that observed in Fig. 2 with channel $\mathbf{H}^{(1)}$. This is mainly due to the relatively weaker interfering link in this channel setup. For example, the interference-to-signal power gain ratio at user 1's receiver is given by $\left|h_{12}\right|^{2} /\left|h_{11}\right|^{2}=0.051$, which is much smaller than 0.53 in channel $\mathbf{H}^{(1)}$. Note that intuitively, it is the non-negligible mutual interference among the users that is exploited by improper Gaussian signaling to outperform the conventional proper Gaussian signaling 3 Therefore, for channel realization $\mathbf{H}^{(2)}$ with almost negligible interfering link for user 1, no observable performance gain can be achieved by

\footnotetext{
${ }^{2}$ The achievable rate region with TS is obtained by taking the convex-hull operation over all the achievable rate-pairs given in 11 .

${ }^{3}$ Consider the extreme case where all the interfering link gains vanish to zero and the SISO-IC reduces to $K$ decoupled Gaussian point-to-point channels. In this case, proper Gaussian signaling is known to be optimal.
} 


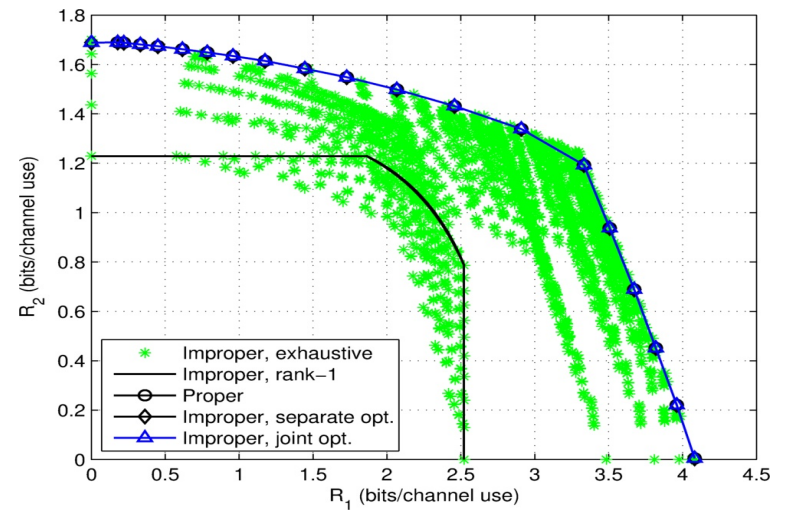

Fig. 4: Achievable rate region for the two-user SISO-IC with channel realization $\mathbf{H}^{(2)}$, and $\mathrm{SNR}=0 \mathrm{~dB}$.

improper Gaussian signaling. Another observation from Fig. 4 is that the rank-1 improper signaling scheme [44], which is based on the equivalent real-valued $2 \times 2$ MIMO-IC, gives strictly smaller rate region than that by proper Gaussian signaling. In contrast, our proposed improper signaling schemes with either joint or separate covariance and pseudo-covariance optimizations, are observed to perform no worse than the optimal proper Gaussian signaling, in accordance with our previous discussion.

\section{Max-Min Rate Comparison}

The rate-profile technique used in characterizing the Pareto boundary of the achievable rate region can be directly applied for maximizing the minimum (max-min) rate of the two users without TS. Specifically, the max-min problem for the twouser SISO-IC is equivalent to solving (P1) by using the rateprofile $\boldsymbol{\alpha}=(1 / 2,1 / 2)$. An alternative max-min solution with improper Gaussian signaling was proposed in [45], where based on the equivalent real-valued $2 \times 2$ MIMO-IC, the transmit covariance matrix of the equivalent real-valued signal vector for each user is assumed to be of rank-1. Note that the use of rank-1 transmission in both [44] and [45] can be justified by the fact that the total DoF of two-user $2 \times 2$ MIMO-ICs exactly equals to 2 [51]. For the ease of precoder design, zero-forcing (ZF) receivers were further applied in [45]. As a benchmark comparison, we also plot the max-min rate achievable by the simple time division multiple access (TDMA) scheme, where for simplicity, each user is assumed to access the channel for half of the time.

To evaluate the average max-min rates, 500 random channel realizations are simulated, where the channel coefficients are drawn from independent zero-mean CSCG random variables. For this example, asymmetric channels are considered, where the average power values of the direct and interfering channels are 1 and 0.2 , respectively, i.e., $h_{k k} \sim \mathcal{C N}(0,1), h_{k \bar{k}} \sim$ $\mathcal{C N}(0,0.2), k=1,2, \bar{k} \neq k$. The obtained results are shown in Fig. 5. The optimal max-min rate achievable by proper Gaussian signaling and that by improper Gaussian signaling obtained by the exhaustive search method [45] are also included in the figure. It is observed that in the low SNR regime, there is no notable gain by improper Gaussian signaling over conventional proper Gaussian signaling, which

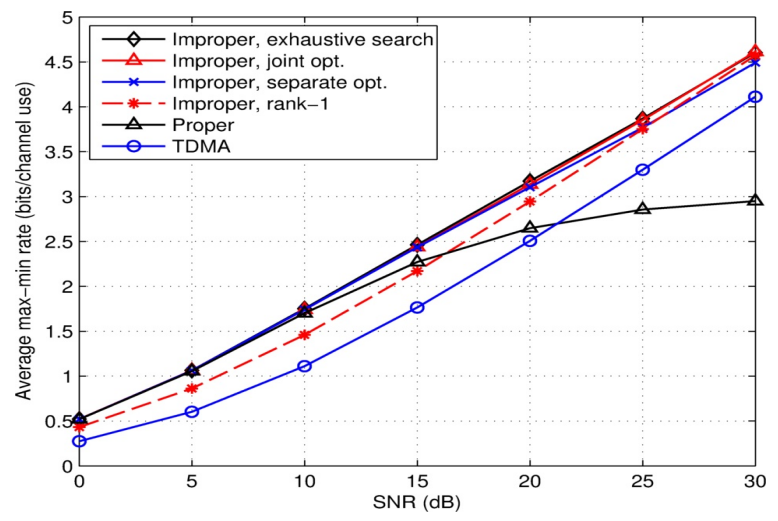

Fig. 5: Average max-min rate for the two-user SISO-IC.

is due to the negligible interference levels at low SNRs. As SNR increases, the max-min rate by proper Gaussian signaling saturates since the total number of data streams transmitted, which is 2, exceeds the total number of DoF of the twouser SISO-IC, which is 1 . In contrast, the linear increase of the max-min rates with respect to the logarithm of SNR can be achieved either by TDMA, or by improper Gaussian signaling. It is worth remarking that over the entire SNR range, the proposed algorithms based on covariance and pseudocovariance optimizations yield close-to-optimal performance obtained by exhaustive search method. On the other hand, the rank-1 transmission with $\mathrm{ZF}$ receivers based on the equivalent real-valued MIMO-IC gives a near-optimal performance in the high-SNR regime, which is expected due to the optimality of ZF receivers at high SNR as well as the DoF optimality of rank-1 transmission as pointed out in [44|45]; however, in the low and moderate SNR regime, the rank-1 transmission scheme results in strictly suboptimal performance, which may be due to the noise enhancement issue associated with ZF receivers applied in [45], as well as the over-conservative number of data streams used by assuming rank-1 transmit covariance matrices.

\section{Sum-Rate Comparison}

In this subsection, the sum-rate maximization with improper Gaussian signaling is considered. By using the equivalent realvalued MIMO-IC of the complex-valued SISO-IC, existing sum-rate maximization algorithms in the literature, such as the one via the iterative weighted MSE minimization (WMMSE) [27], can be applied directly for maximizing the sum-rate of the two-user SISO-IC when improper Gaussian signaling is employed. However, although the WMMSE algorithm is guaranteed to converge to a local maximum of the sum-rate, it is not guaranteed to achieve the global sum-rate maximum. With the algorithms proposed in this paper via covariance and pseudo-covariance optimization, we illustrate with the following example that our proposed algorithms strictly improve the achievable sum-rate over that by the WMMSE algorithm.

In order to apply the WMMSE algorithm [27] to the sumrate maximization problem when improper Gaussian signaling is applied, we transform the complex-valued channel to the equivalent real-valued MIMO channel, similarly as in [43][45]. Denote $\mathbf{Q}_{k}$ as the transmit covariance matrix of user $k$ in the equivalent real-valued $2 \times 2$ MIMO-IC. Without loss 
TABLE I: Equivalence between complex-valued SISO-IC and real-valued MIMO-IC

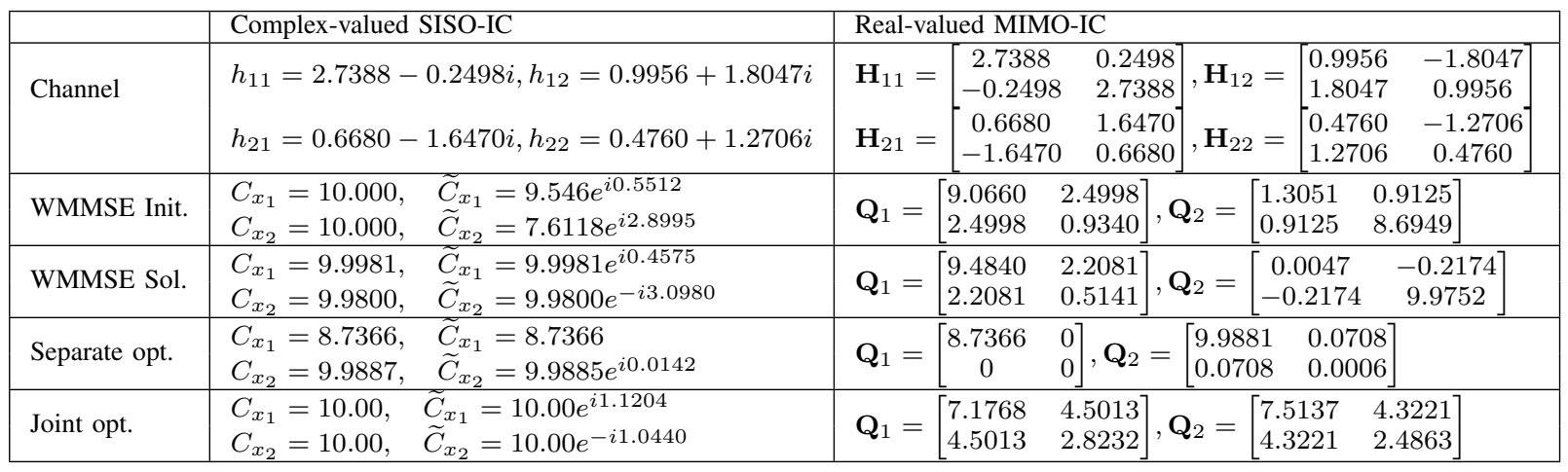

TABLE II: Sum-Rate comparison

\begin{tabular}{|l|c|c|c|}
\hline & WMMSE & Separate opt. & Joint opt. \\
\hline Rate-pair & $(2.8673,1.8102)$ & $(3.4079,2.1515)$ & $(3.4761,2.2078)$ \\
\hline Sum-rate & 4.6775 & 5.5594 & 5.6839 \\
\hline Improvement & - & $18.85 \%$ & $21.52 \%$ \\
\hline
\end{tabular}

of generality, denote the rate-pair obtained by the iterative WMMSE scheme by $r \cdot\left(\delta_{1}, \delta_{2}\right)$, with $\delta_{1} \geq 0, \delta_{2} \geq 0$, and $\delta_{1}+\delta_{2}=1$. With the rate-profile $\boldsymbol{\alpha}=\left(\delta_{1}, \delta_{2}\right)$, (P1) is solved to obtain a new sum-rate $R$. If $R>r$, then the rate-pair obtained by the WMMSE algorithm cannot be sum-rate optimal, and a strictly improved sum-rate can be obtained by our proposed algorithms. Table $\Pi$ shows the obtained sum-rate result. The equivalence of the channel matrices and the converged transmit parameters between the original complex-valued SISO-IC and the equivalent real-valued MIMO-IC for this example is shown in Table I] with the SNR set as $10 \mathrm{~dB}$.

\section{CONCLUSION}

This paper studied the transmit optimization for Gaussian ICs when improper or circularly asymmetric complex Gaussian signaling is applied. Under the assumption that the interference is treated as additive Gaussian noise, it was shown that the use of conventional proper or circularly symmetric complex Gaussian signaling may result in undesired rate loss. A new achievable rate expression for the general MIMO-IC was derived, which is expressed as a summation of the rate achievable with the conventional proper Gaussian signaling, and an additional term due to the use of improper Gaussian signaling. This result provides a useful method to improve the rate over the conventional proper Gaussian signaling by separately optimizing the covariance and pseudo-covariance matrices. We also proposed the technique of widely linear precoding, which efficiently maps the proper Gaussian information-bearing signals to the improper Gaussian transmitted signals with any given pair of covariance and pseudocovariance matrices. Furthermore, for the two-user SISO-IC, we formulated the optimization problem to characterize the Pareto boundary of the achievable rate region via the rateprofile method. Both joint and separate covariance and pseudocovariance optimization algorithms were proposed, both of which outperform the conventional proper Gaussian signaling and provide advantages over existing improper Gaussian signaling schemes.

\section{APPENDIX A \\ Proof of LEMMA 3}

The derivations for (36) and 37) follow similar arguments. For brevity, we only show that of (36) as follows:

$$
\begin{aligned}
\left|\widetilde{C}_{y_{k}}\right|^{2}+\sigma^{4} & \leq\left(\left|\widetilde{C}_{y_{k}}\right|+\sigma^{2}\right)^{2}=\left(\left|h_{k 1}^{2} \widetilde{C}_{x_{1}}+h_{k 2}^{2} \widetilde{C}_{x_{2}}\right|+\sigma^{2}\right)^{2} \\
& \stackrel{(a)}{\leq}\left(\left|h_{k 1}\right|^{2}\left|\widetilde{C}_{x_{1}}\right|+\left|h_{k 2}\right|^{2}\left|\widetilde{C}_{x_{2}}\right|+\sigma^{2}\right)^{2} \\
& \stackrel{(b)}{\leq}\left(\left|h_{k 1}\right|^{2} C_{x_{1}}+\left|h_{k 2}\right|^{2} C_{x_{2}}+\sigma^{2}\right)^{2}=C_{y_{k}}^{2},
\end{aligned}
$$

where $(a)$ follows from the triangle inequality, and $(b)$ is true due to the constraint 35 in (P1.1).

\section{APPENDIX B \\ PROOF OF THEOREM 4}

For notational convenience, in this appendix, we use $X_{1}$ and $X_{2}$ to represent $\widetilde{C}_{x_{1}}$ and $\widetilde{C}_{x_{2}}$, respectively. First, the following proposition shows that to solve (P1.9), we may consider exterior solutions only, i.e., the solutions at which at least one of the inequality constraints is active.

Proposition 1. If $\left\{X_{1}, X_{2}\right\}$ is feasible to (P1.9) with $\left|X_{1}\right|<$ $C_{x_{1}}^{\star}$ and $\left|X_{2}\right|<C_{x_{2}}^{\star}$, then there exists another feasible solution $\left\{X_{1}^{\prime}, X_{2}^{\prime}\right\}$ with $\left|X_{1}^{\prime}\right|=C_{x_{1}}^{\star}$ or $\left|X_{2}^{\prime}\right|=C_{x_{2}}^{\star}$.

Proof: Let $\tau \triangleq \min \left\{\frac{C_{x_{1}}^{\star}}{\left|X_{1}\right|}, \frac{C_{x_{2}}^{\star}}{\left|X_{2}\right|}\right\}$. Then $\tau>1$. Define $X_{1}^{\prime}=\tau X_{1}$ and $X_{2}^{\prime}=\tau X_{2}$. Then the constraints in 64 and (65) are satisfied by $X_{1}^{\prime}$ and $X_{2}^{\prime}$, i.e., $\left|X_{1}^{\prime}\right| \leq C_{x_{1}}^{\star}$ and $\left|X_{2}^{\prime}\right| \leq C_{x_{2}}^{\star}$. Furthermore, at least one of them is satisfied with equality. The constraint in (62) is also satisfied since

$$
\begin{aligned}
& a_{1}\left|h_{11}^{2} X_{1}^{\prime}+h_{12}^{2} X_{2}^{\prime}\right|^{2}+b_{1}=\tau^{2} a_{1}\left|h_{11}^{2} X_{1}+h_{12}^{2} X_{2}\right|^{2}+b_{1} \\
& \stackrel{(a)}{\leq} \tau^{2}\left(a_{1}\left|h_{11}^{2} X_{1}+h_{12}^{2} X_{2}\right|^{2}+b_{1}\right) \stackrel{(b)}{\leq} \tau^{2}\left|X_{2}\right|^{2}=\left|X_{2}^{\prime}\right|^{2},
\end{aligned}
$$

where $(a)$ comes from $\tau>1$ and $b_{1} \geq 0$, and $(b)$ is true since $\left\{X_{1}, X_{2}\right\}$ is feasible to (P1.9). Similarly, (63) is also satisfied. Therefore, $\left\{X_{1}^{\prime}, X_{2}^{\prime}\right\}$ is a feasible solution to (P1.9) with at least one of the inequality constraints being active.

Next, we derive Theorem 4 using the Karush-Kuhn-Tucker (KKT) conditions, which are necessary optimality conditions 
for the constrained optimization problem (P1.9) [50]. For notational convenience, denote the inequality constraints (62)(65) by $f_{1} \leq 0, f_{2} \leq 0, h_{1} \leq 0$ and $h_{2} \leq 0$, respectively. Denote $\lambda_{1}, \lambda_{2}, \mu_{1}, \mu_{2}$ as the corresponding dual variables, respectively. The Lagrangian function of (P1.9) is given by

$$
\begin{aligned}
& L\left(X_{1}, X_{2}, \lambda_{1}, \lambda_{2}, \mu_{1}, \mu_{2}\right)=\lambda_{1}\left\{a _ { 1 } \left(\left|h_{11}\right|^{4} X_{1}^{*} X_{1}+\left|h_{12}\right|^{4} X_{2}^{*} X_{2}\right.\right. \\
& \left.\left.+2 \Re\left\{h_{11}^{2 *} h_{12}^{2} X_{1}^{*} X_{2}\right\}\right)+b_{1}-X_{2}^{*} X_{2}\right\}+\lambda_{2}\left\{a _ { 2 } \left(\left|h_{21}\right|^{4} X_{1}^{*} X_{1}\right.\right. \\
& \left.\left.+\left|h_{22}\right|^{4} X_{2}^{*} X_{2}+2 \Re\left\{h_{21}^{2 *} h_{22}^{2} X_{1}^{*} X_{2}\right\}\right)+b_{2}-X_{1}^{*} X_{1}\right\} \\
& +\mu_{1}\left(X_{1}^{*} X_{1}-C_{x_{1}}^{\star 2}\right)+\mu_{2}\left(X_{2}^{*} X_{2}-C_{x_{2}}^{\star 2}\right) .
\end{aligned}
$$

For $\left\{X_{1}, X_{2}\right\}$ to be a solution to (P1.9), the following KKT conditions must be satisfied:

1) Dual feasibility: $\lambda_{1} \geq 0, \lambda_{2} \geq 0, \mu_{1} \geq 0, \mu_{2} \geq 0$.

2) Zero derivative: The derivatives of the Lagrangian function (69) are zero [52]:

$$
\begin{aligned}
\frac{\partial L}{\partial X_{2}^{*}}=0 \Rightarrow & -X_{2} \underbrace{\left[\left(a_{1}\left|h_{12}\right|^{4}-1\right) \lambda_{1}+\lambda_{2} a_{2}\left|h_{22}\right|^{4}+\mu_{2}\right]}_{\triangleq c_{2}} \\
& =X_{1}(\lambda_{1} \underbrace{a_{1} h_{12}^{2 *} h_{11}^{2}}_{\triangleq V_{1}}+\lambda_{2} \underbrace{a_{2} h_{22}^{2 *} h_{21}^{2}}_{\triangleq V_{2}}) \\
\frac{\partial L}{\partial X_{1}^{*}}=0 \Rightarrow & X_{1} \underbrace{\left[\left(a_{2}\left|h_{21}\right|^{4}-1\right) \lambda_{2}+\lambda_{1} a_{1}\left|h_{11}\right|^{4}+\mu_{1}\right]}_{\triangleq c_{1}} \\
& =-X_{2}(\lambda_{1} \underbrace{a_{1} h_{11}^{2 *} h_{12}^{2}}_{=V_{1}^{*}}+\lambda_{2} \underbrace{a_{2} h_{21}^{2 *} h_{22}^{2}}_{=V_{2}^{*}}), \\
& \Downarrow \\
-X_{2} c_{2} & =X_{1}\left(\lambda_{1} V_{1}+\lambda_{2} V_{2}\right), \\
X_{1} c_{1} & =-X_{2}\left(\lambda_{1} V_{1}^{*}+\lambda_{2} V_{2}^{*}\right) .
\end{aligned}
$$

3) Complementary slackness: $\lambda_{1} f_{1}=0, \lambda_{2} f_{2}=0, \mu_{1} h_{1}=$ $0, \mu_{2} h_{2}=0$.

As discussed previously, without loss of generality, $X_{1}$ can be assumed to be a nonnegative real number. The following cases are then considered to derive the possible phases $\theta$ of $X_{2}$ :

- Case I: $f_{1}=0$ and $f_{2} \neq 0$. Then from the complementary slackness condition, we have $\lambda_{1}>0$ and $\lambda_{2}=0$. Substituting them into (71), we have

$$
X_{2}=-\frac{X_{1}\left(\lambda_{1} a_{1}\left|h_{11}\right|^{4}+\mu_{1}\right)}{\lambda_{1}\left|V_{1}\right|^{2}} V_{1}
$$

Since $\lambda_{1} \geq 0, \mu_{1} \geq 0, a_{1} \geq 0$ and $X_{1} \geq 0$, the phase $\theta$ of $X_{2}$ equals to that of $V_{1}$ rotated by $\pi$, which is $\pi+$ $2\left(\phi_{11}-\phi_{12}\right)$ since $V_{1}=a_{1} h_{12}^{2 *} h_{11}^{2}$.

- Case II: $f_{1} \neq 0$ and $f_{2}=0$. Then $\lambda_{1}=0$ and $\lambda_{2}>0$. Similarly, by using (70), we have $\theta=\pi+2\left(\phi_{21}-\phi_{22}\right)$.

- Case III: $f_{1} \neq 0$ and $f_{2} \neq 0$, then $\lambda_{1}=0$ and $\lambda_{2}=0$. By substituting them into (70) and (71), we have $\mu_{2} X_{2}=0$ and $\mu_{1} X_{1}=0$. If $X_{2}=0$, then $\theta$ can be any arbitrary value. If $X_{1}=0$, then $X_{2}=0$ is implied due to (63) and again, $\theta$ is arbitrary. Therefore, we may focus on $\mu_{1}=0$ and $\mu_{2}=0$ only. However, Proposition 1 suggests that we may consider the exterior solutions only, i.e., either $h_{1}=0$ or $h_{2}=0$ is satisfied. Thus, $\mu_{1}>0$ or $\mu_{2}>0$ can be assumed. Therefore, case III can be ignored without loss of optimality.

- Case IV: $f_{1}=0$ and $f_{2}=0$, then $\theta$ belongs to the solution set for the equations given in 66) and (67), which are obtained by satisfying the constraints $f_{1}$ in 62 and $f_{2}$ in (63) with equality. (66) and (67) correspond to $\left|X_{1}\right|=C_{x_{1}}^{\star}$ and $\left|X_{2}\right|=C_{x_{2}}^{\star}$, respectively, which can be assumed without loss of generality due to Proposition 1 This completes the proof of Theorem 4

\section{APPENDIX C \\ Solving $\Theta_{\mathcal{A}}$ AND $\Theta_{\mathcal{B}}$ IN TheOREM 4}

In this appendix, we show the steps to solve $\Theta_{\mathcal{A}}$, while the solution of $\Theta_{\mathcal{B}}$ can be obtained similarly and thus omitted. Note that the unknown variables in 66 are $\theta$ and $t$. After some manipulations, (66) can be written as

$$
\begin{aligned}
& t \cos \eta+d_{1} t^{2}+d_{2}=0 \\
& t \cos (\eta+\omega)+d_{3} t^{2}+d_{4}=0,
\end{aligned}
$$

where

$$
\begin{aligned}
& \omega \triangleq 2\left(\phi_{22}+\phi_{11}-\phi_{12}-\phi_{21}\right), \eta \triangleq \theta+2\left(\phi_{12}-\phi_{11}\right), \\
& d_{1} \triangleq \frac{a_{1}\left|h_{12}\right|^{4}-1}{2 a_{1}\left|h_{11}\right|^{2}\left|h_{12}\right|^{2} C_{x_{1}}^{\star}}, d_{2} \triangleq \frac{a_{1}\left|h_{11}\right|^{4} C_{x_{1}}^{\star 2}+b_{1}}{2 a_{1}\left|h_{11}\right|^{2}\left|h_{12}\right|^{2} C_{x_{1}}^{\star}}, \\
& d_{3} \triangleq \frac{\left|h_{22}\right|^{2}}{2\left|h_{21}\right|^{2} C_{x_{1}}^{\star}}, \quad d_{4}=\frac{\left(a_{2}\left|h_{21}\right|^{4}-1\right) C_{x_{1}}^{\star 2}+b_{2}}{2 a_{2}\left|h_{21}\right|^{2}\left|h_{22}\right|^{2} C_{x_{1}}^{\star}} .
\end{aligned}
$$

From (73), we have

$$
\begin{aligned}
& t \sin \eta \sin \omega=t \cos \eta \cos \omega+d_{3} t^{2}+d_{4} \Rightarrow \\
& t^{2}\left(1-\cos ^{2} \eta\right) \sin ^{2} \omega=\left(t \cos \eta \cos \omega+d_{3} t^{2}+d_{4}\right)^{2}
\end{aligned}
$$

Solving $\cos \eta$ from (72), we have

$$
\cos \eta=-\left(d_{1} t^{2}+d_{2}\right) / t \text {. }
$$

Substituting (76) into (75) gives the following fourth order polynomial equation with respect to $t$ :

$\left[t^{2}-\left(d_{1} t^{2}+d_{2}\right)^{2}\right] \sin ^{2} \omega=\left[\left(d_{3}-d_{1} \cos \omega\right) t^{2}+d_{4}-d_{2} \cos \omega\right]^{2}$. Since the above equation only has terms involving $t^{2}$, it can be transformed to the following quadratic equation by setting $z=t^{2}$, i.e.,

$$
e_{1} z^{2}+e_{2} z+e_{3}=0
$$

where $e_{1}=d_{3}^{2}+d_{1}^{2}-2 d_{1} d_{3} \cos \omega$,

$$
\begin{aligned}
& e_{2}=2\left(d_{1} d_{2}+d_{3} d_{4}\right)-2\left(d_{1} d_{4}+d_{2} d_{3}\right) \cos \omega-\sin ^{2} \omega, \\
& e_{3}=d_{2}^{2}+d_{4}^{2}-2 d_{2} d_{4} \cos \omega .
\end{aligned}
$$

Then $z$ can be easily solved. Since $z=t^{2}$ and $t$ is the magnitude of $\widetilde{C}_{x_{2}}$, only the solutions of $z$ that are real and satisfy $0 \leq z \leq C_{x_{2}}^{\star 2}$ need to be kept, whereby the values for $t$ are obtained. For those values of $t$ satisfying $\left|\left(d_{1} t^{2}+d_{2}\right) / t\right| \leq 1$, we can get the value for $\eta$ based on (76), i.e., $\eta=\arccos \left[-\left(d_{1} t^{2}+d_{2}\right) / t\right]$ or $\eta=2 \pi-\arccos \left[-\left(d_{1} t^{2}+\right.\right.$ $\left.d_{2}\right) / t$. Then $\theta$ can be obtained from (74). If no such solutions exist, then $\Theta_{\mathcal{A}}$ is set to empty.

\section{REFERENCES}

[1] A. B. Carleial, "A case where interference does not reduce capacity," IEEE Trans. Inf. Theory, vol. 21, no. 5, pp. 569-570, Sep. 1975.

[2] T. S. Han and K. Kobayashi, "A new achievable rate region for the interference channel," IEEE Trans. Inf. Theory, vol. 27, no. 1, pp. 4960, Jan. 1981.

[3] R. Etkin, D. Tse, and H. Wang, "Gaussian interference channel capacity to within one bit," IEEE Trans. Inf. Theory, vol. 54, no. 1, pp. 55345562, Dec. 2008

[4] X. Shang, G. Kramer, and B. Chen, "A new outer bound and noisyinterference sum-rate capacity for the Gaussian interference channels," IEEE Trans. Inf. Theory, vol. 55, no. 2, pp. 689-699, Feb. 2009. 
[5] M. Chiang, P. Hande, T. Lan, and C. W. Tan, Power Control in Wireless Cellular Networks. Foundations and Trends in Networking, 2008.

[6] A. Gjendemsjø, D. Gesbert, G. E. Øien, and S. G. Kiani, "Binary power control for sum rate maximization over multiple interfering links," IEEE Trans. Wireless Commun., vol. 7, no. 8, pp. 3164-3173, Aug. 2008.

[7] Z.-Q. Luo and S. Zhang, "Dynamic spectrum management: complexity and duality," IEEE J. Sel. Topics Signal Process., vol. 2, no. 1, pp. 5773, Feb. 2008

[8] F. R. Farrokhi, K. J. R. Liu, and L. Tassiulas, "Transmit beamforming and power control for cellular wireless systems," IEEE J. Sel. Areas Commun., vol. 16, no. 8, pp. 1437-1450, Oct. 1998.

[9] E. Visotsky and U. Madhow, "Optimal beamforming using transmit antenna arrays,", in Proc. IEEE Veh. Technol. Conf., vol. 1, Houston, Texas, May 1999, pp. 851-856.

[10] M. Schubert and H. Boche, "Solution of the multiuser downlink beamforming problem with individual SINR constraints," IEEE Trans. Veh. Technol., vol. 53, no. 1, pp. 18-28, Jan. 2004.

[11] H. Dahrouj and W. Yu, "Coordinated beamforming for the multicell multi-antenna wireless system," IEEE Trans. Wireless Commun., vol. 9, no. 5 , pp. $1748-1759$, May 2010

[12] B. Song, R. Cruz, and B. Rao, "Network duality for multiuser MIMO beamforming networks and applications," IEEE Trans. Commun. vol. 55, no. 3, pp. 618-630, Mar. 2007.

[13] A. Wiesel, Y. C. Eldar, and S. Shamai (Shitz), "Linear precoding via conic optimization for fixed MIMO receivers," IEEE Trans. Signal Process., vol. 54, no. 1, pp. 161-176, Jan. 2006.

[14] M. Bengtsson and B. Ottersten, "Optimal downlink beamforming using semidefinite optimization," in Proc. 37th Allerton Conf. on Commun.,Control, and Computing, Mar. 1999, pp. 987-996.

[15] Y. F. Liu, Y. H. Dai, and Z.-Q. Luo, "Coordinated beamforming for MISO interference channel: complexity analysis and efficient algorithms," IEEE Trans. Signal Process., vol. 59, no. 3, pp. 1142-1156, Mar. 2011.

[16] J. Huang, R. A. Berry, and M. L. Honig, "Distributed interference compensation for wireless networks," IEEE J. Sel. Areas Commun., vol. 24, no. 5, pp. 1074-1084, May 2006.

[17] R. Zakhour and D. Gesbert, "Coordination on the MISO interference channel using the virtual SINR framework," in Proc. ITG/IEEE Workshop Smart Antennas, Feb. 2009.

[18] S. Ye and R. S. Blum, "Optimized signaling for MIMO interference systems with feedback," IEEE Trans. Signal Process., vol. 51, no. 11 , pp. 2839-2848, Nov. 2003.

[19] H. Sung, K. J. Lee, S. H. Park, and I. Lee, "Linear precoder designs for $K$-user interference channels," IEEE Trans. Wireless Commun., pp. 291-301, Jan. 2010

[20] L. P. Qian, Y. J. Zhang, and J. Huang, "MAPEL: achieving global optimality for a non-convex wireless power control problem," IEEE Trans. Wireless Commun., vol. 8, no. 3, pp. 1553-1563, Mar. 2009.

[21] E. A. Jorswieck and E. G. Larsson, "Monotonic optimization framework for the two-user miso interference channel," IEEE Trans. Commun., vol. 58, no. 7, pp. 2159-2168, Jul. 2010

[22] L. Liu, R. Zhang, and K. C. Chua, "Achieving global optimality for weighted sum-rate maximization in the $K$-user Gaussian interference channel with multiple antennas," IEEE Trans. Wireless Commun., vol. 11, no. 5, pp. 1933-1945, May 2012.

[23] W. Utschick and J. Brehmer, "Monotonic optimization framework for coordinated beamforming in multicell networks," IEEE Trans. Signal Process., vol. 60, no. 4, pp. 1899-1909, Apr. 2012.

[24] E. Björnson, G. Zheng, M. Bengtsson, and B. Ottersten, "Robust monotonic optimization framework for multicell MISO systems," IEEE Trans. Signal Process., vol. 60, no. 5, pp. 2508-2523, May 2012.

[25] S. S. Christensen, R. Agarwal, E. D. Carvalho, and J. M. Cioffi, "Weighted sum-rate maximization using weighted MMSE for MIMOBC beamforming design," IEEE Trans. Wireless Commun., vol. 7, no. 12, pp. 4792-4799, Dec. 2008 .

[26] M. Razaviyayn, M. Sanjabi, and Z.-Q. Luo, "Linear transceiver design for interference alignment: complexity and computation," IEEE Trans. Inf. Theory, vol. 58, no. 5, pp. 2896-2910, May 2012.

[27] Q. Shi, M. Razaviyayn, Z.-Q. Luo, and C. He, "An iteratively weighted MMSE approach to distributed sum-utility maximization for a MIMO interfering broadcast channel," IEEE Trans. Signal Process., vol. 59, no. 9, pp. 4331-4340, Sep. 2011.

[28] G. Scutari, P. Palomar, and S. Barbarossa, "Competitive design of multiuser MIMO systems based on game theory: a unified view," IEEE J. Sel. Areas Commun., vol. 26, no. 7, pp. 1089-1103, Sep. 2008.
[29] A. Host-Madsen and A. Nosratinia, "The multiplexing gain of wireless networks," Int. Symp. on Inf. Theory and Its Applications, pp. 20652069, 4-9 Sept. 2005.

[30] V. R. Cadambe and S. A. Jafar, "Interference alignment and degrees of freedom of the $K$-user interference channel," IEEE Trans. Inf. Theory, vol. 54, no. 8, pp. 3425-3441, Aug. 2008.

[31] S. W. Peters and R. W. Heath Jr., "Cooperative algorithms for MIMO interference channels," IEEE Trans. Veh. Technol., vol. 60, no. 1, pp. 206 - 218, Jan. 2011

[32] D. A. Schmidt, C. Shi, R. A. Berry, M. L. Honig, and W. Utschick, "Minimum mean squared error interference alignment," IEEE Asilomar Conference on Signals, Systems and Computers (ACSSC), pp. 1106 1110, Nov. 2009.

[33] K. Gomadam, V. R. Cadambe, and S. A. Jafar, "A distributed numerical approach to interference alignment and applications to wireless interference networks," IEEE Trans. Inf. Theory, vol. 57, no. 6, pp. 3309-3322, May 2011

[34] M. Charafeddine, A. Sezgin, and A. Paulraj, "Rates region frontiers for $n$-user interference channel with interference as noise," in Proc. Allerton Conference, Sep. 2007.

[35] E. Jorswieck, E. Larsson, and D. Danev, "Complete characterization of the Pareto boundary for the MISO interference channel," IEEE Trans. Signal Process., vol. 56, no. 10, pp. 5292-5296, Oct. 2008

[36] R. Zhang and S. Cui, "Cooperative interference management with MISO beamforming," IEEE Trans. Signal Process., vol. 58, no. 10, pp. 54505458 , Oct. 2010.

[37] X. Shang, B. Chen, and H. V. Poor, "Multiuser MISO interference channles with single-user detection: optimality of beamforming and the achievable rate region," IEEE Trans. Inf. Theory, vol. 57, no. 7, pp. 4255 - 4273, Jul. 2011.

[38] R. Mochaourab and E. A. Jorswieck, "Optimal beamforming in interference networks with perfect local channel information," IEEE Trans. Signal Process., vol. 59, no. 3, pp. 1128-1141, Mar. 2011.

[39] F. D. Neeser and J. L. Massey, "Proper complex random processes with applications to information theory," IEEE Trans. Inf. Theory, vol. 39, no. 4, pp. 1293 - 1302, Jul. 1993

[40] P. J. Schreier and L. L. Scharf, Statistical Signal Processing of ComplexValued Data: The Theory of Improper and Noncircular Signals. Cambridge (UK): Cambridge Univ. Press, 2010.

[41] G. Tauböck, "Complex-valued random vectors and channels: entropy, divergence, and capacity," IEEE Trans. Inf. Theory, vol. 58, no. 5, pp. 2729-2744, May 2012.

[42] S. A. Jafar, Interference Alignment: A New Look at Signal Dimensions in a Communication Network. Foundations and Trends in Communications and Information Theory, 2010, vol. 7, no. 1.

[43] V. R. Cadambe, S. A. Jafar, and C. Wang, "Interference alignment with asymmetric complex signaling - settling the Host-Madsen-Nosratinia conjecture," IEEE Trans. Inf. Theory, pp. 4552 - 4565, Sep. 2010.

[44] Z. K. M. Ho and E. Jorswieck, "Improper Gaussian signaling on the two-user SISO interference channel," IEEE Trans. Wireless Commun., vol. 11, no. 9, pp. 3194 - 3203, Sep. 2012.

[45] S. H. Park, H. Park, and I. Lee, "Coordinated SINR balancing techniques for multi-cell downlink transmission," in Proc. VTC 2010-fall, 2010.

[46] P. J. Schreier and L. L. Scharf, "Second-order analysis of improper complex random vectors and processes," IEEE Trans. Signal Process., vol. 51, no. 3, pp. 714-725, Mar. 2003.

[47] Z.-Q. Luo, W. K. Ma, A. M. So, Y. Ye, and S. Zhang, "Semidefinite relaxation of quadratic optimization problems," IEEE Signal Processing Mag, vol. 27, no. 3, pp. 20-34, May 2010.

[48] R. A. Horn and C. R. Johnson, Matrix Analysis, 2nd ed. New York: Cambridge Univ. Press, 2013.

[49] B. Picinbono and P. Chevalier, "Widely linear estimation with complex data," IEEE Trans. Signal Process., vol. 43, no. 8, pp. 2030-2033, Aug. 1995.

[50] S. Boyd and L. Vandenberghe, Convex Optimization. Cambridge, U.K.: Cambridge Univ. Press, 2004.

[51] S. A. Jafar and M. Fakhereddin, "Degrees of freedom for the MIMO interference channel," IEEE Trans. Inf. Theory, vol. 53, no. 7, pp. 2637 2642, Jul. 2007.

[52] A. Hjørungnes, Complex-Valued Matrix Derivatives With Applications in Signal Processing and Communications. Cambridge (UK): Cambridge Univ. Press, 2011. 